\title{
Infinite Systems of Functional Equations and Gaussian Limiting Distributions
}

\author{
Michael Drmota 邦 Bernhard Gittenberger 啡 and Johannes F. Morgenbesser非
}

${ }^{1}$ Institut für Diskrete Mathematik und Geometrie, TU Wien, Austria

${ }^{2}$ Fakultät für Mathematik, Universität Wien, Austria

In this paper infinite systems of functional equations in finitely or infinitely many random variables arising in combinatorial enumeration problems are studied. We prove sufficient conditions under which the combinatorial random variables encoded in the generating function of the system tend to a finite or infinite dimensional limiting distribution.

Keywords: generating functions, functional equation, singularity analysis, central limit theorem

\section{Introduction}

Systems of functional equations for generating functions appear in many combinatorial enumeration problems, for example in tree enumeration problems or in the enumeration of planar graphs (and related problems), see Drmota (2009). Usually, these enumeration techniques can be extended to take several parameters into account: the number of vertices, the number of edges, the number of vertices of a given degree etc.

One of the simplest examples is that of rooted plane trees, that are defined as rooted trees, where each node has an arbitrary number of successors with a natural left-to-right-order. By splitting up at the root one obtains a recursive description of rooted plane trees (see Figure 1) which translates into corresponding relations for the counting generating function $y(x)=\sum_{n \geq 1} y_{n} x^{n}$ :

$$
y(x)=x+x y(x)+x y(x)^{2}+x y(x)^{3}+\cdots=\frac{x}{1-y(x)} .
$$

Of course, this leads to

$$
y(x)=\frac{1-\sqrt{1-4 x}}{2}
$$

and to

$$
y_{n}=\frac{1}{n}\left(\begin{array}{c}
2 n-2 \\
n-1
\end{array}\right) .
$$

\footnotetext{
${ }^{\dagger}$ Email: michael.drmota@tuwien. ac.at. Supported by the Austrian Science Foundation, Project S9604.

¥Email: bernhard.gittenberger@tuwien. ac . at. Supported by the Austrian Science Foundation, Project S9604.

$\S$ Email: johannes.morgenbesser@univie.ac.at. Supported by the Austrian Science Foundation, Projects S9604 and P21209.
} 
Now let $\mathbf{k}=\left(k_{0}, k_{1}, k_{2}, \ldots\right)$ be a sequence of non-negative integers and $y_{n, \mathbf{k}}$ the number of rooted plane trees with $n$ vertices such that $k_{j}$ vertices have exactly $j$ successors (that is, the out-degree equals $j$ ) for all $j \geq 0$. Then the formal generating function $y(x, \mathbf{u})=\sum_{n, \mathbf{k}} y_{n, \mathbf{k}} x^{n} \mathbf{u}^{\mathbf{k}}$, where $\mathbf{u}=\left(u_{0}, u_{1}, u_{2}, \ldots\right)$ and $\mathbf{u}^{\mathbf{k}}=u_{0}^{k_{0}} u_{1}^{k_{1}} u_{2}^{k_{2}} \cdots$, satisfies the equation

$$
y(x, \mathbf{u})=x u_{0}+x u_{1} y(x, \mathbf{u})+x u_{2} y(x, \mathbf{u})^{2}+x u_{3} y(x, \mathbf{u})^{3}+\cdots=F(x, y(x, \mathbf{u}), \mathbf{u}) .
$$

If $\|\mathbf{u}\|_{\infty}$ is bounded then this can be considered as an analytic equation for $y(x, \mathbf{u})$, and of course $y(x, \mathbf{u})$ encodes the distribution of the number of vertices of given out-degree. More precisely, sup-

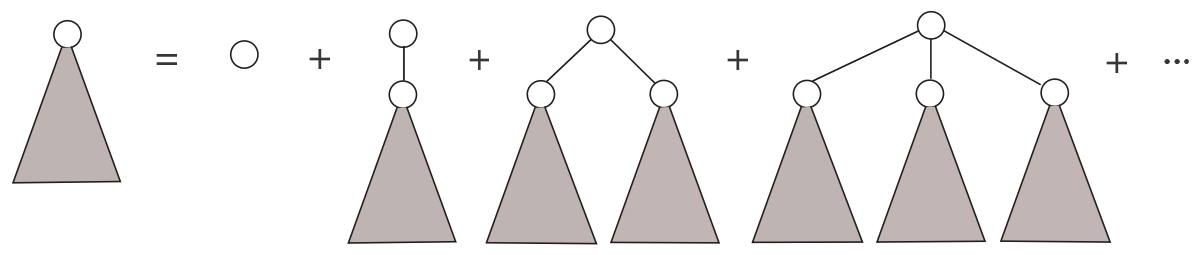

Fig. 1: Recursive structure of a rooted plane tree

pose that all rooted plane trees of size $n$ are equally likely. Then the number of vertices with outdegree $j$ becomes a random variable $X_{n}^{(j)}$. If we now consider the infinite dimensional random vector $\mathbf{X}_{n}=\left(X_{n}^{(0)}, X_{n}^{(1)}, X_{n}^{(2)}, \ldots\right)$ then we have in this uniform random model

$$
\mathbb{E} \mathbf{u}^{\mathbf{X}_{n}}=\frac{1}{y_{n}}\left[x^{n}\right] y(x, \mathbf{u}),
$$

where $\left[x^{n}\right] y(x)$ denotes the coefficient of $x^{n}$ in the series expansion of $y(x)$. Let $\ell$ be a linear functional of the form $\ell \cdot \mathbf{X}_{n}=\sum_{j \geq 0} s_{j} X_{n}^{(j)}$ then we also have

$$
\mathbb{E} e^{i t \ell \cdot \mathbf{X}_{n}}=\frac{1}{y_{n}}\left[x^{n}\right] y\left(x, e^{i t s_{0}}, e^{i t s_{1}}, \ldots\right) .
$$

This also means that the asymptotic behavior of the characteristic function of $\ell \cdot \mathbf{X}_{n}$ (that determines the limiting distribution) can be derived from the asymptotic behavior of $\left[x^{n}\right] y(x, \mathbf{u})$. In this way it follows (more or less) by standard methods that $X_{n}^{(j)}$ and also all finite dimensional random vectors $\left(X_{n}^{(0)}, X_{n}^{(1)}, \ldots, X_{n}^{(K)}\right)$ satisfy a (finite) dimensional central limit theorem. Nevertheless it is not immediately clear that the infinite random vector $\mathbf{X}_{n}$ has Gaussian limiting distribution, too. (For a definition of infinite dimensional Gaussian distributions see Section 2.) In Theorem 3 we will give a sufficient condition for such a property when the generating function $y(x, \mathbf{u})$ satisfies a single functional equation $y(x, \mathbf{u})=F(x, y(x, \mathbf{u}), \mathbf{u})$.

In more refined enumeration problems it will be necessary to replace the (single) equation for $y(x, \mathbf{u})$ by a finite or infinite system of equations $\mathbf{y}=\mathbf{F}(x, \mathbf{y}, \mathbf{u})$; see Section 4. More precisely, this means that we have to split up our enumeration problem into finitely or infinitely many subproblems that are interrelated. If $y_{i}$ denotes the generating function of the $i$-th subproblem then this means that $y_{i}(x, \mathbf{u})=$ $F_{i}(x, \mathbf{y}(x, \mathbf{u}), \mathbf{u})$ for a certain function $F_{i}$. After having solved this system of equations the generating 
function $y(x, \mathbf{u})$ for the original problem can be computed with the help of the generating functions $y_{i}$, that is $y(x, \mathbf{u})=G(x, \mathbf{y}(x, \mathbf{u}), \mathbf{u})$ for a properly chosen function $G$.

In this case we are faced with two different problems. First of all a system of equations is more difficult to solve than a single equation, in particular in the infinite dimensional case. However, this can be handled by assuming compactness of the Jacobian of the system, see Theorem 1 Furthermore it turns out that the problem on the infinite dimensional Gaussian distribution is even more involved than in the single equation case. Nevertheless we will prove that all bounded functionals $\ell \cdot \mathbf{X}_{n}$ have a Gaussian limiting distribution which is a slightly weaker result, see Theorem 2

The structure of the paper is as follows. In Section 2 we collect some facts from functional analysis that are needed to formulate our main results that are stated in Section 3 . The corresponding proofs can be found in the Appendix whereas some applications are given in Section 4

Finally we would like to mention that this paper is a continuation of the work of Drmota (1997) and Morgenbesser (2010).

\section{Preliminaries}

Before we state the main result, we recall some definitions from the field of functional analysis in order to be able to specify the basic setting. Let $B_{1}$ and $B_{2}$ be Banach spaces. We denote by $L\left(B_{1}, B_{2}\right)$ the set of bounded linear operators from $B_{1}$ to $B_{2}$. If $U$ is the open unit ball in $B_{1}$, then an operator $T: B_{1} \rightarrow B_{2}$ is compact, if the closure of $T(U)$ is compact in $B_{2}$ (or, equivalently, if every bounded sequence $\left(x_{n}\right)_{n \geq 0}$ in $B_{1}$ contains a subsequence $\left(x_{n_{i}}\right)_{i \geq 0}$ such that $\left(T x_{n_{i}}\right)_{i \geq 0}$ converges in $\left.B_{2}\right)$. If $A$ is a bounded operator from $B$ to $B$, then $r(A)$ denotes the spectral radius of $A$ defined by $r(A)=\sup _{\lambda \in \sigma(A)}|\lambda|$, where $\sigma(A)$ is the spectrum of $A$.

A function $F: B_{1} \rightarrow B_{2}$ is called Fréchet differentiable at $x_{0}$ if there exists a bounded linear operator $(\partial F / \partial x)\left(x_{0}\right): B_{1} \rightarrow B_{2}$ such that

$$
F\left(x_{0}+h\right)=F\left(x_{0}\right)+\frac{\partial F}{\partial x}\left(x_{0}\right) h+\omega\left(x_{0}, h\right) \quad \text { and } \quad \omega\left(x_{0}, h\right)=o(\|h\|), \quad(h \rightarrow 0) .
$$

The operator $\partial F / \partial x$ is called the Fréchet derivative of $F$. If the Banach spaces are complex vector spaces and (3) holds for all $h$, then $F$ is said to be analytic in $x_{0} . F$ is analytic in $D \subseteq B_{1}$, if it is analytic for all $x_{0} \in D$. Analyticity is equivalent to the fact that for all $x_{0} \in D$ there exist an $s>0$ and continuous symmetric $n$-linear forms $A_{n}\left(x_{0}\right)$ such that $\sum_{n \geq 1}\left\|A_{n}\left(x_{0}\right)\right\| s^{n}<\infty$ and

$$
F\left(x_{0}+h\right)=F\left(x_{0}\right)+\sum_{n \geq 1} \frac{A_{n}\left(x_{0}\right)}{n !}\left(h^{n}\right)
$$

in a neighborhood of $x_{0}$ (including the set $\left\{x_{0}+h:\|h\| \leq s\right\}$ ). (The "coefficients" $A_{n}$ are equal to the (iteratively defined) $n$-th Fréchet derivatives of $F$ ). See for example (Deimling, 1985, Section 7.7 and 15.1), (Zeidler, 1986. Chapters 4 and 8) and (Reich and Shoikhet, 2005, Chapter 2) for analytic functions in Banach spaces.

Next, we want to recall some facts concerning probability theory on Banach spaces (see for example Billingsley (1999); Ledoux and Talagrand (1991)). Suppose that $\mathbf{X}$ is a random variable from a probability space $(\Omega, \mathcal{F}, \mathbb{P})$ (here, $\Omega$ denotes a set with $\sigma$-algebra $\mathcal{F}$ and probability measure $\mathbb{P}$ ) to a separable Banach space $B$ (equipped With the Borel $\sigma$-algebra). Let $P$ be the law (the distribution) of $\mathbf{X}$ (that 
is, $P=\mathbb{P} \mathbf{X}^{-1}$ ). Since we assumed $B$ to be separable, we have that the scalar valued random variables $\ell^{*}(\mathbf{X})$ for continuous functionals $\ell^{*}$ determine the distribution of $\mathbf{X}$ (see (Ledoux and Talagrand, 1991 , Section 2.1)).

The random variables $\mathbf{X}_{n}, n \in \mathbb{N}$ (with possibly different probability spaces) are said to converge weakly to some $B$-valued random variable $\mathbf{X}$ (defined on some probability space and with law $P$ ) if the corresponding laws $P_{n}$ converge weakly to $P$, i.e., if we have (as $n$ goes to infinity)

$$
\int_{B} f \mathrm{~d} P_{n} \rightarrow \int_{B} f \mathrm{~d} P
$$

for every bounded continuous real function $f$. In what follows we denote this by $\mathbf{X}_{n} \stackrel{w}{\rightarrow} \mathbf{X}$. We call a set $\Pi$ of probability measures tight if for each $\varepsilon>0$ there exists a compact set $K=K_{\varepsilon}$ such that $P(K)>1-\varepsilon$ for every $P \in \Pi$. Let $B^{*}$ be the dual space of $B$ (the set of continuous functionals from $B$ to $\mathbb{C}$ ). By Prohorov's theorem (see (Billingsley, 1999, Chapter I, Section 5)) we have that $\mathbf{X}_{n}$ weakly converges to $\mathbf{X}$ if and only if $\ell^{*}\left(\mathbf{X}_{n}\right)$ weakly converges to $\ell^{*}(\mathbf{X})$ for all $\ell^{*} \in B^{*}$ and the family of probability measures $\left\{P_{n}: n \in \mathbb{N}\right\}$ is tight. (Prohorov's theorem says that in a separable and complete metric space a set of probability measures is tight if and only if it is relatively compact.) Since for scalar valued random variables the weak convergence is completely determined by the convergence of the corresponding characteristic functions, one has to check

(i) tightness of the set $\left\{P_{n}: n \in \mathbb{N}\right\}$

and

(ii) there exists an $\mathbf{X}$ such that $\mathbb{E}\left[e^{i t \ell^{*}\left(\mathbf{X}_{n}\right)}\right] \rightarrow \mathbb{E}\left[e^{i t \ell^{*}(\mathbf{X})}\right]$ for all $\ell^{*} \in B^{*}$,

in order to show $\mathbf{X}_{n} \stackrel{w}{\rightarrow} \mathbf{X}$. We call a random variable $\mathbf{X}$ Gaussian if $\ell^{*}(\mathbf{X})$ is a Gaussian variable (in the extended sense that $X \equiv 0$ is also normally distributed) for all $\ell^{*} \in B^{*}$. If it exists, we denote by $\mathbb{E} \mathbf{X}$ the (unique) element $\mathbf{y} \in B$ such that

$$
\ell^{*}(\mathbf{y})=\mathbb{E}\left(\ell^{*}(\mathbf{X})\right)
$$

for all $\ell^{*} \in B^{*}$. Gaussian variables are called centered, if $\mathbb{E} \mathbf{X}=\mathbf{0}$.

In what follows, we mainly deal with the Banach space $\ell^{p}=\ell^{p}(\mathbb{N})(1 \leq p<\infty)$ of all complex valued sequences $\left(t_{n}\right)_{n \in \mathbb{N}}$ satisfying $\left\|\left(t_{n}\right)\right\|_{p}^{p}:=\sum_{n=1}^{\infty}\left|t_{n}\right|^{p}<\infty$. (The space $\ell^{\infty}=\ell^{\infty}(\mathbb{N})$ is the space of all bounded complex sequences $\left(z_{n}\right)$ with norm $\left\|\left(z_{n}\right)\right\|_{\infty}=\sup _{n \geq 1}\left|z_{n}\right|<\infty$.) In this case, the Fréchet derivative is also called Jacobian operator (in analogy to the finite dimensional case). We call a function $\mathbb{F}: \mathbb{C} \times \ell^{p} \rightarrow \ell^{p}$ positive (in $U \times V$ ), if there exist nonnegative real numbers $a_{i \mathbf{j}, k}$ such that for all $k \geq 1$ and for all $(x, \mathbf{y}) \in U \times V$,

$$
F_{k}(x, \mathbf{y})=\sum_{i, \mathbf{j}} a_{i \mathbf{j}, k} x^{i} y^{\mathbf{j}}
$$

where $\mathbf{j} \in \mathbb{N}^{\mathbb{N}}$, only finitely many components are nonzero, and $y^{\mathbf{j}}=y_{1}^{j_{1}} y_{2}^{j_{2}} y_{3}^{j_{3}} \cdots$.

In our main theorem we have to assume that $\partial F / \partial \mathbf{y}$ is irreducible. In order to be able to define this property, we recall some basic notion from functional analysis on $\ell^{p}$ spaces. Any bounded linear operator 
on an $\ell^{p}$ space $(1 \leq p<\infty)$ is uniquely determined by an infinite dimensional matrix $\left(a_{i j}\right)_{1 \leq i, j<\infty}$ via the functional

$$
(A x)_{i}=\sum_{k=1}^{\infty} a_{i k} x_{k},
$$

where $\left(x_{k}\right)_{1 \leq k<\infty}$ is written with respect to the canonical standard bases in $\ell^{p}$. We call the matrix $\left(a_{i j}\right)_{1 \leq i, j<\infty}$ the matrix representation of $A$ (and write $A=\left(a_{i j}\right)_{1 \leq i, j<\infty}$ or just $A=\left(a_{i j}\right)$ ). An operator $A$ is called positive, if all entries of the matrix representation of $A$ are nonnegative. A positive operator $A=\left(a_{i j}\right)$ is said to be irreducible, if for every pair $(i, j)$ there exists an integer $n=n(i, j)>0$, such that $a_{i j}^{(n)}>0$, where

$$
A^{n}=\left(a_{i j}^{(n)}\right)_{1 \leq i, j<\infty} .
$$

If $u$ and $v$ are real vectors or matrices, $u \geq v$ means that all entries of $u$ are greater than or equal to the corresponding entries of $v$. Thus, an operator $A$ is positive if $\left(a_{i j}\right) \geq 0$. Similarly, a vector $x$ is called positive (or also nonnegative) if $x \geq 0$. We call $x$ strictly positive, if all entries $x_{i}$ of $x$ satisfy $x_{i}>0$. Moreover, if $u$ is a vector with entries $u_{i}$, then $|u|$ denotes the vector with entries $\left|u_{i}\right|$ (a corresponding definition is used for matrices).

The dual space of $\ell^{p}, 1<p<\infty$ is isomorphic to $\ell^{q}$, where $1 / p+1 / q=1$. Note, that the dual space of $\ell^{1}$ is $\ell^{\infty}$. If $p$ is fixed, we use throughout this work the letter $q$ for the real number which satisfies $1 / p+1 / q=1$ if $p>1$ and $q=\infty$ if $p=1$. If $x \in \ell^{p}$ and $\ell \in \ell^{q} \cong\left(\ell^{p}\right)^{\prime}$, we denote by $\ell(x)$ the functional $\ell$ evaluated at $x$. Analogous to the finite dimensional case, we also use the notation $\ell \cdot x$ and $\ell^{T} x$ instead of $\ell(x)$.

If $1<p<\infty$, the adjoint operator of an operator $A$ (denoted by $A^{*}$ ) is acting on $\ell^{p^{\prime}} \cong \ell^{q}$. The operator $A^{*}$ can be associated with the matrix $\left(a_{j i}\right)_{1 \leq i, j<\infty}$ acting on $\ell^{q}$ (which we do in the sequel without explicitly saying so). If $x$ is an eigenvector of $A$ we also call it right eigenvector of $A$ and if $y$ is an eigenvector of $A^{*}$ we call it left eigenvector of $A$.

The study of operators (or matrices) in $\ell^{\infty}$ is different. In fact, the space $\ell^{\infty}$ is not separable and there is no one-to-one correspondence between operators and matrices. (Actually, there exist nontrivial compact operators, such that the corresponding "matrix representation" is the zero matrix.) Nevertheless, if we have a matrix $\left(a_{i j}\right)_{1 \leq i, j<\infty}$, we define an operator $A$ on $\ell^{\infty}$ via

$$
(A x)_{i}=\sum_{k=1}^{\infty} a_{i k} x_{k},
$$

if the summation is well-defined for all $i \geq 1$ and for all $x \in \ell^{\infty}$. In the case that $A=\left(a_{i j}\right)_{1 \leq i, j<\infty}$ is an operator from $\ell^{1}$ to $\ell^{1}$, we get that the dual operator from $\ell^{\infty}$ to $\ell^{\infty}$ is given by $\left(a_{j i}\right)_{1 \leq i, j<\infty}$ (as in the $\ell^{p}$-case for $p>1$ ).

Throughout, we denote by $\mathrm{I}_{\mathrm{p}}$ the identity operator on $\ell^{p}$ (with matrix representation $\left(\delta_{i j}\right)_{1 \leq i, j<\infty}$, where $\delta_{i j}$ denotes Kronecker's delta function).

\section{Main Theorems}

Our first result is a generalization of a result of Morgenbesser (2010), where only one counting variable was considered. It determines the kind of singularity of the solution of a positive irreducible and infinite 
system of equations. Note that it is more convenient to write $\mathbf{u}$ in the form $\mathbf{u}=e^{\mathbf{v}}$, that is, $u_{j}=e^{v_{j}}$. The reason is that in the functional analytic context of our results it is natural to work in a neighborhood of $\mathbf{v}=\mathbf{0}$ instead of a neighborhood of $\mathbf{u}=\mathbf{1}$. Anyway, in the applications we will use again $\mathbf{u}$ since this is more natural for counting problems.

Theorem 1 Let $1 \leq p<\infty, 1 \leq r \leq \infty$ and $\mathbf{F}: \mathbb{C} \times \ell^{p} \times \ell^{r} \rightarrow \ell^{p},(x, \mathbf{y}, \mathbf{v}) \mapsto \mathbf{F}(x, \mathbf{y}, \mathbf{v})$ be a function satisfying:

1. there exist open balls $B \in \mathbb{C}, U \in \ell^{p}$ and $V \in \ell^{r}$ such that $(0, \mathbf{0}, \mathbf{0}) \in B \times U \times V$ and $\mathbf{F}$ is analytic in $B \times U \times V$,

2. the function $(x, \mathbf{y}) \mapsto \mathbf{F}(x, \mathbf{y}, \mathbf{0})$ is a positive function,

3. $\mathbf{F}(0, \mathbf{y}, \mathbf{v})=\mathbf{0}$ for all $\mathbf{y} \in U$ and $\mathbf{v} \in V$,

4. $\mathbf{F}(x, \mathbf{0}, \mathbf{v}) \not \equiv \mathbf{0}$ in $B$ for all $\mathbf{v} \in V$,

5. $\frac{\partial \mathbf{F}}{\partial \mathbf{y}}(x, \mathbf{y}, \mathbf{0})=A(x, \mathbf{y})+\alpha(x, \mathbf{y}) \mathrm{I}_{\mathrm{p}}$ for all $(x, \mathbf{y}) \in B \times U$, where $\alpha$ is an analytic function and there exists an integer $n$ such that $A^{n}$ is compact,

6. $A(x, \mathbf{y})$ is irreducible for strictly positive $(x, \mathbf{y})$ and $\alpha(x, \mathbf{y})$ has positive Taylor coefficients.

Let $\mathbf{y}=\mathbf{y}(x, \mathbf{v})$ be the unique solution of the functional equation

$$
\mathbf{y}=\mathbf{F}(x, \mathbf{y}, \mathbf{v})
$$

with $\mathbf{y}(0, \mathbf{v})=\mathbf{0}$. Assume that for $\mathbf{v}=\mathbf{0}$ the solution has a finite radius of convergence $x_{0}>0$ such that $\mathbf{y}_{0}:=\mathbf{y}\left(x_{0}, \mathbf{0}\right)$ exists and $\left(x_{0}, \mathbf{y}_{0}\right) \in B \times U$.

Then there exists $\varepsilon>0$ such that $\mathbf{y}(x, \mathbf{v})$ admits a representation of the form

$$
\mathbf{y}(x, \mathbf{v})=\mathbf{g}(x, \mathbf{v})-\mathbf{h}(x, \mathbf{v}) \sqrt{1-\frac{x}{x_{0}(\mathbf{v})}}
$$

for $\mathbf{v}$ in a neighborhood of $\mathbf{0},\left|x-x_{0}(\mathbf{v})\right|<\varepsilon$ and $\arg \left(x-x_{0}(\mathbf{v})\right) \neq 0$, where $\mathbf{g}(x, \mathbf{v}), \mathbf{h}(x, \mathbf{v})$ and $x_{0}(\mathbf{v})$ are analytic functions with $\mathbf{h}_{i}\left(x_{0}(\mathbf{0}), \mathbf{0}\right)>0$ for all $i \geq 1$.

Moreover, if there exist two integers $n_{1}$ and $n_{2}$ that are relatively prime such that $\left[x^{n_{1}}\right] \mathbf{y}_{1}(x, \mathbf{0})>0$ and $\left[x^{n_{2}}\right] \mathbf{y}_{1}(x, \mathbf{0})>0$, then $x_{0}(\mathbf{v})$ is the only singularity of $\mathbf{y}(x, \mathbf{v})$ on the circle $|x|=x_{0}(\mathbf{v})$ and there exist constants $0<\delta<\pi / 2$ and $\eta>0$ such that $\mathbf{y}(x, \mathbf{v})$ is analytic in a region of the form

$$
\Delta:=\left\{x:|x|<x_{0}(\mathbf{0})+\eta, \mid \arg \left(x / x_{0}(\mathbf{v})-1\right)>\delta\right\} .
$$

Remark 1 As we will show in the proof of Theorem 1 the point $\left(x_{0}, \mathbf{y}_{0}\right)$ satisfies the equations

$$
\begin{aligned}
& \mathbf{y}_{0}=\mathbf{F}\left(x_{0}, \mathbf{y}_{0}, \mathbf{0}\right), \\
& r\left(\frac{\partial \mathbf{F}}{\partial \mathbf{y}}\left(x_{0}, \mathbf{y}_{0}, \mathbf{0}\right)\right)=1 .
\end{aligned}
$$

The main reason for this property is the fact that we have assumed that $\left(x_{0}, \mathbf{y}_{0}\right)$ lies in the domain of analyticity of F. For a detailed study in the finite dimensional case of such so called critical points see Bell] et al. (2010). Note furthermore, that the existence of a point $\left(x_{0}, \mathbf{y}_{0}\right)$ satisfying the above equations implies that $\mathbf{F}$ is a nonlinear function in $\mathbf{y}$. 
Remark 2 In the stated result we have assumed that the function $F$ is defined on some subset of $\mathbb{C} \times$ $\ell^{p} \times \ell^{r}$, where $1 \leq p<\infty$ and $1 \leq r \leq \infty$ and that the range of $F$ is a subset of $\ell^{p}$. The same result (with obvious modifications of the proof) holds true if one replaces one (or both) of the spaces $\ell^{p}$ and $\ell^{r}$ by finite dimensional spaces $\mathbb{R}^{m}$ and $\mathbb{R}^{n}$. In the case that both spaces are replaced by finite dimensional ones, the statement was proven in Drmota (1997).

Corollary 1 Let $\mathbf{y}=\mathbf{y}(x, \mathbf{v})$ be the unique solution of the functional equation (4) and assume that all assumptions of Theorem 1 are satisfied. Suppose that $G:\left(\mathbb{C}, \ell^{p}, \ell^{r}\right) \rightarrow \mathbb{C}$ is an analytic function such that $\left(x_{0}(\mathbf{0}), \mathbf{y}\left(x_{0}(\mathbf{0}), \mathbf{0}\right), \mathbf{0}\right)$ is contained in the interior of the region of convergence and that

$$
\frac{\partial G}{\partial \mathbf{y}}\left(x_{0}(\mathbf{0}), \mathbf{y}\left(x_{0}(\mathbf{0}), \mathbf{0}\right), \mathbf{0}\right) \neq \mathbf{0} .
$$

Then there exists $\delta, \varepsilon>0$ such that $G(x, \mathbf{y}(x, \mathbf{v}), \mathbf{v})$ has a representation of the form

$$
G(x, \mathbf{y}(x, \mathbf{v}), \mathbf{v})=\bar{g}(x, \mathbf{v})-\bar{h}(x, \mathbf{v}) \sqrt{1-\frac{x}{x_{0}(\mathbf{v})}}
$$

for $\left|x-x_{0}(\mathbf{v})\right| \leq \varepsilon$ and $\arg \left(x-x_{0}(\mathbf{0})\right) \neq 0$ and for $\mathbf{v}$ in a neighborhood of $\mathbf{0}$. The functions $\bar{g}(x, \mathbf{v})$, $\bar{h}(x, \mathbf{v})$ and $x_{0}(\mathbf{v})$ are analytic in this domain and $\bar{h}\left(x_{0}(\mathbf{0}), \mathbf{0}\right) \neq 0$. Moreover, $G(x, \mathbf{y}(x, \mathbf{v}), \mathbf{v})$ is analytic for $\mathbf{v}$ in a neighborhood of $\mathbf{0}$ and $\left|x-x_{0}(\mathbf{v})\right| \geq \varepsilon$ but $|x| \leq\left|x_{0}(\mathbf{v})\right|+\eta$ and we have

$$
\left[x^{n}\right] G(x, \mathbf{y}(x, \mathbf{v}), \mathbf{v})=\frac{\bar{h}\left(x_{0}(\mathbf{v}), \mathbf{v}\right)}{2 \sqrt{\pi}} x_{0}(\mathbf{v})^{-n} n^{-3 / 2}\left(1+O\left(\frac{1}{n}\right)\right)
$$

uniformly for $\mathbf{v}$ in a neighborhood of $\mathbf{0 .}$

As mentioned in the introduction, the solution of a finite or infinite system of equations, $\mathbf{y}(x, \mathbf{v})$, is used to represent the generating function $y(x, \mathbf{v})$ of the original combinatorial problem, that is $y(x, \mathbf{v})=$ $G(x, \mathbf{y}(x, \mathbf{v}), \mathbf{v})$. If we write it as

$$
y(x, \mathbf{v})=G(x, \mathbf{y}(x, \mathbf{v}), \mathbf{v})=\sum_{n=0}^{\infty} c_{n}(\mathbf{v}) x^{n},
$$

(where $\mathbf{y}(x, \mathbf{v})$ satisfies a functional equation $\mathbf{y}=\mathbf{F}(x, \mathbf{y}, \mathbf{v})$ with $\mathbf{y}(0, \mathbf{v})=\mathbf{0}$ such that the assumptions of Theorem 1 are satisfied) and $\mathbf{X}_{n}$ denotes the corresponding $\ell^{p}$-valued random variable defined on some probability space $(\Omega, \mathcal{F}, \mathbb{P})(1 \leq p<\infty)$ then

$$
\mathbb{E}\left[e^{i t \boldsymbol{\ell} \cdot \mathbf{X}_{n}}\right]=\frac{c_{n}(i t \boldsymbol{\ell})}{c_{n}(\mathbf{0})}
$$

for all $\ell \in \ell^{q}$. In applications one can think of $G(x, \mathbf{y}, \mathbf{v})$ to be of the form

$$
G(x, \mathbf{y}(x, \mathbf{v}), \mathbf{v})=\sum_{n=0}^{\infty} \sum_{\mathbf{m} \in \ell^{p}} c_{n, \mathbf{m}} e^{\mathbf{m} \cdot \mathbf{v}} x^{n}=\sum_{n=0}^{\infty} \sum_{\mathbf{m} \in \ell^{p}} c_{n, \mathbf{m}} \mathbf{u}^{\mathbf{m}} x^{n}
$$

where $c_{n, \mathbf{m}}$ denotes the number of objects of size $n$ and characteristic $m$. Our second result shows that all bounded functionals of $\mathbf{X}_{n}$ satisfy a central limit theorem. 
Theorem 2 Let $1 \leq p<\infty$ and suppose that $\mathbf{X}_{n}$ is a sequence of $\ell^{p}$-valued random variables defined by (7). Furthermore, let $\ell \in \ell^{q}$. Then we have $\ell \cdot \mathbb{E} \mathbf{X}_{n}=\mu_{\ell} n+O(1)$ with $\mu_{\ell}=-\frac{\partial x_{0}}{\partial \mathbf{v}}(\mathbf{0}) \cdot \ell / x_{0}$ and

$$
\ell \cdot\left(\frac{\mathbf{X}_{n}-\mathbb{E} \mathbf{X}_{n}}{\sqrt{n}}\right)
$$

weakly converges for $n$ to infinity to a centered real Gaussian variable with variance $\sigma_{\ell}^{2}=\ell^{T} B \boldsymbol{\ell}$, where $B \in L\left(\ell^{q}, \ell^{p}\right)$ is given by the matrix

$$
\frac{1}{x_{0}^{2}}\left(\frac{\partial x_{0}}{\partial \mathbf{v}_{i}}(\mathbf{0}) \cdot \frac{\partial x_{0}}{\partial \mathbf{v}_{j}}(\mathbf{0})^{T}\right)_{1 \leq i, j<\infty}-\frac{1}{x_{0}}\left(\frac{\partial^{2} x_{0}}{\partial \mathbf{v}_{i} \mathbf{v}_{j}}(\mathbf{0})\right)_{1 \leq i, j<\infty} .
$$

Corollary 2 Let $1 \leq p<\infty$ and suppose that $\mathbf{X}_{n}$ is a sequence of $\ell^{p}$-valued random variables defined by (7) such that the set of laws of $\left(\mathbf{X}_{n}-\mathbb{E} \mathbf{X}_{n}\right) / \sqrt{n}, n \geq 1$ is tight. Then there exists a centered Gaussian random variable $\mathbf{X}$ such that

$$
\frac{\mathbf{X}_{n}-\mathbb{E} \mathbf{X}_{n}}{\sqrt{n}} \stackrel{w}{\rightarrow} \mathbf{X}
$$

where $\mathbf{X}$ is uniquely determined by the operator $B \in L\left(\ell^{q}, \ell^{p}\right)$ stated in Theorem 2

It is clear that we cannot expect tightness in all cases. For example if we have

$$
y(x, \mathbf{v})=x e^{\sum_{j \geq 0} v_{j}}+\frac{x y(x, \mathbf{v})}{1-y(x, \mathbf{v})}
$$

then all random variables $X_{n}^{(j)}(j \geq 0)$ count the number of leaves in rooted plane trees and the sequence $\left(\mathbf{X}_{n}-\mathbb{E} \mathbf{X}_{n}\right) / \sqrt{n}$ is not tight. Actually, the next theorem shows that even in the case of a single equation we have to check several non-trivial assumptions. It is far from being obvious how these properties might generalize to the general case.

Theorem 3 Suppose that $y(x, \mathbf{v})$ is the unique solution of a single functional equation $y=F(x, y, \mathbf{v})$, where $F: B \times U \times V \rightarrow \mathbb{C}$ is a positive analytic function on $B \times U \times V \subseteq \mathbb{C}^{2} \times \ell^{2}$ such that there exist positive real $\left(x_{0}, y_{0}\right) \in B \times U$ with $y_{0}=F\left(x_{0}, y_{0}, \mathbf{0}\right)$ and $1=F_{y}\left(x_{0}, y_{0}, \mathbf{0}\right)$ such that $F_{x}\left(x_{0}, y_{0}, \mathbf{0}\right) \neq 0$ and $F_{y y}\left(x_{0}, y_{0}, \mathbf{0}\right) \neq 0$. Furthermore assume that the corresponding random variables $X_{n}^{(j)}$ have the property that $X_{n}^{(j)}=0$ if $j>$ cn for some constant $c>0$ and that the following conditions are satisfied:

$$
\begin{gathered}
\sum_{j \geq 0} F_{v_{j}}<\infty, \quad \sum_{j \geq 0} F_{y v_{j}}^{2}<\infty, \quad \sum_{j \geq 0} F_{v_{j} v_{j}}<\infty, \\
F_{x v_{j}}=o(1), \quad F_{x v_{j} v_{j}}=o(1), \quad F_{y y v_{j}}=o(1), \quad F_{y y v_{j} v_{j}}=o(1), \\
F_{x x v_{j}}=O(1), \quad F_{x y v_{j}}=O(1), \quad F_{x y y v_{j}}=O(1), \quad F_{y y y v_{j}}=O(1), \quad(j \rightarrow \infty)
\end{gathered}
$$

where all derivatives are evaluated at $\left(x_{0}, y_{0}, \mathbf{0}\right)$.

Then the the set of laws of $\left(\mathbf{X}_{n}-\mathbb{E} \mathbf{X}_{n}\right) / \sqrt{n}, n \geq 1$ is tight and has a Gaussian limit.

Finally, we mention the (simpler) case when the function $\mathbf{F}$ is linear in $\mathbf{y}$ (as noted in Remark 1 , we considered until now only the nonlinear case). We just state and prove the following result from which one can deduce corresponding asymptotic expansions of the coefficients and limit theorems. 
Theorem 4 Let $1 \leq p<\infty, 1 \leq r \leq \infty$ and $\mathbf{F}: \mathbb{C} \times \ell^{p} \times \ell^{r} \rightarrow \ell^{p}$, $(x, \mathbf{y}, \mathbf{v}) \mapsto \mathbf{F}(x, \mathbf{y}, \mathbf{v})$ be a linear function in $\mathbf{y}$ satisfying the assumptions (i) -(vi) of Theorem 1 . Set $\mathbf{F}(x, \mathbf{y}, \mathbf{v})=\mathbf{L}(x, \mathbf{v}) \mathbf{y}+\mathbf{b}(x, \mathbf{v})$ and let $\mathbf{y}=\mathbf{y}(x, \mathbf{v})$ be the solution of the functional equation

$$
\mathbf{y}=\mathbf{L}(x, \mathbf{0}) \mathbf{y}+\mathbf{b}(x, \mathbf{v})
$$

with $\mathbf{y}(0, \mathbf{v})=\mathbf{0}$. Assume that there exists a positive number $x_{0}>0$ in the domain of analyticity of $\mathbf{L}(x, \mathbf{v})$ such that

$$
r\left(\mathbf{L}\left(x_{0}, \mathbf{0}\right)\right)=1
$$

Then there exists $\varepsilon>0$ such that $\mathbf{y}(x, \mathbf{v})$ admits a representation of the form

$$
\mathbf{y}(x, \mathbf{v})=\frac{1}{1-\frac{x}{x_{0}(\mathbf{v})}} \mathbf{f}(x, \mathbf{v})
$$

for $\mathbf{v}$ in a neighborhood of $\mathbf{0},\left|x-x_{0}(\mathbf{v})\right|<\varepsilon$ and $\arg \left(x-x_{0}(\mathbf{v})\right) \neq 0$, where $\mathbf{f}(x, \mathbf{v})$ and $x_{0}(\mathbf{v})$ are analytic functions with $\mathbf{f}_{i}\left(x_{0}(\mathbf{0}), \mathbf{0}\right) \neq 0$ for all $i \geq 1$.

\section{Applications}

\subsection{Rooted Plane Trees}

As in the Introduction we consider rooted plane trees, where we also count the number of vertices with out-degree $j \geq 0$. It is clear that the functional equation (2) satisfies the assumptions of Theorem 3 (recall that $\left.x_{0}=1 / 4\right)$. Consequently the random vector $\mathbf{X}_{n}=\left(X_{n}^{(j)}\right)_{j \geq 0}$ satisfies a central limit theorem. The convergence of the finite-dimensional projections (without tightness) was already shown in Pittel (1999).

\subsection{Bipartite Planar Maps}

Planar maps are connected graphs that are embedded on the sphere. Rooted (and also pointed) maps can be counted by several techniques (for example by the quadratic method etc.). Recently, a bijection between rooted maps and so-called mobiles has been established that makes the situation much more transparent, see Bouttier et al. (2004). We restrict ourselves to the case of bipartite maps, that is, all faces have an even degree.

In particular let $R(x, \mathbf{u})$ denote the generating function that solves the equation

$$
R=x+\sum_{j \geq 1} u_{j}\left(\begin{array}{c}
2 j-1 \\
j
\end{array}\right) R^{j} .
$$

Then the generating function $M(x, \mathbf{u})$ of bipartite maps, where $x$ counts the number of edges and $u_{j}$ the number of faces of degree $2 j$ for $j \geq 1$, satisfies $M_{x}=R$.

Here we can also apply Theorem 3 (in this case $x_{0}=1 / 8$ ). Furthermore, since Eulerian maps are dual to bipartite maps we also get a central limit theorem for the degree distribution of Eulerian maps. 


\subsection{Subcritical Graphs}

Every connected graph can be decomposed into 2-connected components (we just have to cut at cut points). Suppose that we are considering a class of connected vertex labeled graphs with the property that all 2-connected components of are also in this class. Let $B(x)$ denote the exponential generating function of 2-connected graphs in this class and $C(x)$ the exponential generating function of all (connected) graphs in this class. By the unique decomposition property we have the relation ( $c f$. (Harary and Palmer, 1973, p.10, (1.3.3)))

$$
C^{\prime}(x)=e^{B^{\prime}\left(x C^{\prime}(x)\right)}
$$

Note that $C^{\prime}(x)$ is the generating function of pointed graphs, that is, one vertex is distinguished (and not labeled). A graph class is called subcritical if the radius of convergence of $B(x)$ is larger than $\eta$, where $\eta$ is defined by the equation $\eta B^{\prime \prime}(\eta)=1$. It has been already proved in Drmota et al. (2011) that the number of vertices of degree $j$ in subcritical graph classes satisfy a central limit theorem. For fixed $j$ it is sufficient to consider just a finite system of equations so that one can apply the methods of Drmota (1997) to obtain the central limit theorem. However, if we want to consider all $j \geq 1$ at once then we are forced to use an infinite system.

Suppose that $B_{r}^{\bullet}\left(x, u_{1}, u_{1}, \ldots\right)$ denotes the generating function of pointed 2-connected graphs, where the pointed vertex has degree $r$ and the variables $x$ and $u_{j}$ count the number of remaining vertices and the (remaining) vertices of degree $j, j \geq 1$. Similarly we define $C_{j}^{\bullet}\left(x, u_{1}, u_{2}, \ldots\right)$ for connected graphs. Then the unique decomposition property implies that we the generating functions satisfy the relations

$$
C_{j}(x, \mathbf{u})=\sum_{l_{1}+2 l_{2}+\cdots j l_{j}=j} \prod_{r=1}^{j} \frac{B_{r}^{\bullet}\left(x, W_{1}, W_{2}, \ldots\right)^{l_{r}}}{l_{r} !},
$$

where $W_{j}$ abbreviates

$$
W_{j}=\sum_{i \geq 0} u_{i+j} C_{i}^{\bullet}(x, \mathbf{u})
$$

with the convention $C_{0}^{\bullet}=1$ (see Drmota et al. (2011)). The generating function of interest is then

$$
C^{\bullet}(x, \mathbf{u})=\sum_{j \geq 0} C_{j}^{\bullet}(x, \mathbf{u}) .
$$

This means that we are actually in the framework of Theorems 1 and 2 The only condition that cannot be directly checked is the compactness condition of the Jacobian. However, we can apply the following general property (that is satisfied in the present example).

Lemma 1 Let $H(x, y, w)$ be a positive functions (as in Theorem 1 in the one dimensional setting) and suppose that $y(x)$ has a finite radius of convergence $x_{0}$ (so that $H(x, y, 1)$ is analytic at $\left(x_{0}, y_{0}\right)$ ) and satisfies the functional equation $y(x)=H(x, y(x), 1)$. Furthermore consider the system of equations

$$
y_{j}(x, \mathbf{u})=F_{j}(x, \mathbf{y}(x, \mathbf{u}), \mathbf{u})
$$

with positive functions that satisfy all assumptions of Theorem 1 except 5. (the compactness of the Jacobian) and where $F_{i}$ has the additional property that

$$
F_{i}(x, \mathbf{y}, \mathbf{1})=\left[w^{i}\right] H\left(x, \sum_{j} y_{j}, w\right) .
$$


Then we have $y(x)=\sum_{i} y_{i}(x, \mathbf{1})$ so that all functions $y_{i}(x, \mathbf{1})$ have the same radius of convergence as $y(x)$ and the operator $A=\frac{\partial \mathbf{F}}{\partial \mathbf{y}}(x, y, \mathbf{1})$ is compact.

Proof: The assumptions imply that $y(x)=\sum_{i} y_{i}(x, \mathbf{1})$ and that $A$ has rank one.

\subsection{Pattern in Trees}

In Chyzak et al. (2008) it is proved that the number of occurrences of a specific pattern in random trees satisfies a central limit theorem. The proof of this result falls precisely into the framework of the present paper. However, it is sufficient to consider a finite system of equations. The combinatorial method of Chyzak et al. (2008) can be naturally extended to count (at once) the number of occurrences of any pattern. Of course, this leads to an infinite system of equations for which Theorems 1 and 2 apply.

\section{Acknowledgements}

The authors express their gratitude to Svante Janson and Steven P. Lalley for many stimulating discussions on the subject.

\section{References}

J. P. Bell, S. N. Burris, and K. A. Yeats. Counting rooted trees: the universal law $t(n) \sim C \rho^{-n} n^{-3 / 2}$. Electron. J. Combin., 13(1):Research Paper 63, 64 pp. (electronic), 2006.

J. P. Bell, S. N. Burris, and K. A. Yeats. Characteristic points of recursive systems. Electron. J. Combin., 17(1):Research Paper 121, 34 pp. (electronic), 2010.

P. Billingsley. Convergence of probability measures. Wiley Series in Probability and Statistics: Probability and Statistics. John Wiley \& Sons Inc., New York, second edition, 1999. A Wiley-Interscience Publication.

J. Bouttier, P. Di Francesco, and E. Guitter. Planar maps as labeled mobiles. Electron. J. Combin., 11(1): Research Paper 69, 27 pp. (electronic), 2004.

F. Chyzak, M. Drmota, T. Klausner, and G. Kok. The distribution of patterns in random trees. Comb. Prob. Computing, 17:21-59, 2008.

K. Deimling. Nonlinear functional analysis. Springer-Verlag, Berlin, 1985.

M. Drmota. Systems of functional equations. Random Structures Algorithms, 10(1-2):103-124, 1997. Average-case analysis of algorithms (Dagstuhl, 1995).

M. Drmota. Random trees. SpringerWienNewYork, Vienna, 2009. An interplay between combinatorics and probability.

M. Drmota, E. Fusy, M. Kang, V. Kraus, and J. Rue. Asymptotic study of subcritical graph classes. SIAM J. Dicrete Maths, 25:1615-1651, 2011. 
P. Flajolet and A. Odlyzko. Singularity analysis of generating functions. SIAM J. Discrete Math., 3(2): 216-240, 1990.

P. Flajolet and R. Sedgewick. Analytic combinatorics. Cambridge University Press, Cambridge, 2009.

U. Grenander. Probabilities on algebraic structures. John Wiley \& Sons Inc., New York, 1963.

F. Harary and E. M. Palmer. Graphical enumeration. Academic Press, New York, 1973.

T. Kato. Perturbation theory for linear operators. Die Grundlehren der mathematischen Wissenschaften, Band 132. Springer-Verlag New York, Inc., New York, 1966.

M. G. Kreı̆n and M. A. Rutman. Linear operators leaving invariant a cone in a Banach space. Amer. Math. Soc. Translation, 1950(26):128, 1950.

S. P. Lalley. Finite range random walk on free groups and homogeneous trees. Ann. Probab., 21(4): 2087-2130, 1993.

S. P. Lalley. Random walks on regular languages and algebraic systems of generating functions. In Algebraic methods in statistics and probability (Notre Dame, IN, 2000), volume 287 of Contemp. Math., pages 201-230. Amer. Math. Soc., Providence, RI, 2001.

S. P. Lalley. Random walks on infinite free products and infinite algebraic systems of generating functions. Preprint, http://www.stat.uchicago.edu/ lalley/Papers/index.html, 2002.

M. Ledoux and M. Talagrand. Probability in Banach spaces, volume 23 of Ergebnisse der Mathematik und ihrer Grenzgebiete (3) [Results in Mathematics and Related Areas (3)]. Springer-Verlag, Berlin, 1991. Isoperimetry and processes.

J. F. Morgenbesser. Square root singularities of infinite systems of functional equations. In 21st International Meeting on Probabilistic, Combinatorial, and Asymptotic Methods in the Analysis of Algorithms (AofA'10), Discrete Math. Theor. Comput. Sci. Proc., AM, pages 513-525. Assoc. Discrete Math. Theor. Comput. Sci., Nancy, 2010.

B. Pittel. Normal convergence problem? Two moments and a recurrence may be the clues. Ann. Appl. Probab., 9(4):1260-1302, 1999. ISSN 1050-5164. doi: 10.1214/aoap/1029962872. URL http: //dx.doi.org/10.1214/aoap/1029962872.

S. Reich and D. Shoikhet. Nonlinear semigroups, fixed points, and geometry of domains in Banach spaces. Imperial College Press, London, 2005.

D. Vere-Jones. Ergodic properties of nonnegative matrices. I. Pacific J. Math., 22:361-386, 1967.

A. R. Woods. Coloring rules for finite trees, and probabilities of monadic second order sentences. Random Structures Algorithms, 10(4):453-485, 1997.

E. Zeidler. Nonlinear functional analysis and its applications. I. Springer-Verlag, New York, 1986. Fixed-point theorems, Translated from the German by Peter R. Wadsack. 


\section{Appendix}

\section{Auxiliary results}

In this section we prove some spectral properties of compact and positive operators on $\ell^{p}$ spaces and we show that the spectral radius of the Jacobian operator of $\mathbf{F}$ (under the assumptions stated in Theorem 1) is continuous.

Recall that the spectrum of a compact operator is a countable set with no accumulation point different from zero. Moreover, each nonzero element from the spectrum is an eigenvalue with finite multiplicity (see for example (Kato, 1966, Chapter III, $\S$ 6.7)). The following result is a generalization of the PerronFrobenius theorem on nonnegative matrices and goes back to Kreĭn and Rutman (1950) (see (Zeidler. 1986, Proposition 7.26)).

Lemma 2 Let $T=\left(t_{i j}\right)_{1 \leq i, j<\infty}$ be a compact positive operator on $\ell^{p}$ (where $1 \leq p<\infty$ ) and assume that $r(T)>0$. Then $r(T)$ is an eigenvalue of $T$ with nonnegative eigenvector $x \in \ell^{p}$. Moreover, $r(T)=r\left(T^{*}\right)$ is an eigenvalue of $T^{*}$ with nonnegative eigenvector $y \in \ell^{q}$.

Lemma 3 Let $A_{1}$ be a positive and irreducible operator on $\ell^{p}$ (where $1 \leq p<\infty$ ) such that $A_{1}^{n}$ is compact for some integer $n \geq 1$. Furthermore let $\alpha \geq 0$ be a real number and set $A=A_{1}+\alpha \mathrm{I}_{\mathrm{p}}$. Then we have $r\left(A_{1}\right)>0$ and $r(A)=r\left(A_{1}\right)+\alpha$ is an eigenvalue of $A$ with strictly positive right eigenvector $x \in \ell^{p}$ and strictly positive left eigenvector $y \in \ell^{q}$.

Proof: First we show that $r\left(A_{1}\right)>0$. Since $A_{1}$ is irreducible, there exists an integer $m$ such that

$$
d=\left(A_{1}^{m}\right)_{11}>0 .
$$

Then we have $\left\|A_{1}^{m n}\right\| \geq d^{n}$ for all $n \geq 1$, where $\|\cdot\|$ denotes the operator norm that is induced by the $p$-norm on $\ell^{p}$ (consider $A_{1}^{m} e_{1}$, where $e_{1}=(1,0,0, \ldots)$ ). Gelfand's formula implies $r(A)=$ $\lim _{n \rightarrow \infty}\left\|A_{1}^{n}\right\|^{1 / n} \geq d^{1 / m}$. Since

$$
\sigma\left(A_{1}^{n}\right)=\left(\sigma\left(A_{1}\right)\right)^{n}
$$

we have that $r:=r\left(A_{1}\right)$ is equal to $r\left(A_{1}^{n}\right)^{1 / n}$. Lemma2 2 implies that $r^{n}$ is an eigenvalue of $A_{1}^{n}$ and there exist vectors $\tilde{x} \in \ell^{p}$ and $\tilde{y} \in \ell^{q}$ such that

$$
A_{1}^{n} \tilde{x}=r^{n} \tilde{x}, \quad \text { and } \quad \tilde{y} A_{1}^{n}=r^{n} \tilde{y} .
$$

Thus we have that $r$ is also in the spectrum of $A_{1}$ and $r(A)=r\left(A_{1}\right)+\alpha>0$. (Note, that $\sigma(A)=$ $\sigma\left(A_{1}\right)+\alpha$.) In the following we show that

$$
x:=\sum_{i=0}^{n-1} r^{i} A_{1}^{n-1-i} \tilde{x}
$$

is a strictly positive right eigenvector of $A_{1}$ to the eigenvalue $r$. It is easy to see that $A_{1} x=r x$. We clearly have that $x$ is nonnegative and $x \neq 0$. Thus, there exists an index $j$ such that $x_{j}>0$. Let $k \geq 1$. Since $A_{1}$ is irreducible, there exists an integer $m$ such that $\left(A_{1}^{m}\right)_{k j}>0$. Since $A_{1}^{m} x=r^{m} x$, we obtain

$$
x_{k}=\frac{1}{r^{m}}\left(A_{1}^{m} x\right)_{k}=\frac{1}{r^{m}} \sum_{\ell=1}^{\infty}\left(A_{1}^{m}\right)_{k \ell} x_{\ell}>\frac{1}{r^{m}}\left(A_{1}^{m}\right)_{k j} x_{j}>0 .
$$


Furthermore, one can show the same way that $y:=\sum_{i=0}^{n-1} r^{i} \tilde{y} A_{1}^{n-1-i}$ is a strictly positive left eigenvector of $A_{1}$ to the eigenvalue $r$.

Proposition 1 Let $1 \leq p<\infty$ and $A=A_{1}+\alpha \mathrm{I}_{\mathrm{p}}, C=C_{1}+\gamma \mathrm{I}_{\mathrm{p}}$ be operators on $\ell^{p}$ with $\alpha \in \mathbb{R}^{+}, \gamma \in$ $\mathbb{C}$ and such that there exists an integer $n$ such that $A_{1}^{n}$ and $C_{1}^{n}$ are compact. Furthermore let $A_{1}$ be positive and irreducible such that $\left|C_{1}\right| \leq A_{1}$ and $|\gamma| \leq \alpha$ but $\left|C_{1}\right|+\left|\gamma \mathrm{I}_{\mathrm{p}}\right| \neq A$. Then we have

$$
r(C)<r(A) .
$$

Proof: Lemma3 3 implies that $r(A) \geq r\left(A_{1}\right)>0$. If $r\left(C_{1}\right)=0$, we have $r(C)=|\gamma|$ and

$$
r(A)=r\left(A_{1}\right)+\alpha>\alpha \geq|\gamma|=r(C) .
$$

Assume now that $r(C)>0$. Since $C_{1}^{n}$ is compact, there exists an eigenvector $z \in \ell^{p}$ to some eigenvalue $s$ with $|s|=r\left(C_{1}\right)$. Since $r(C) \leq r\left(C_{1}\right)+|\gamma|$, we get

$$
r(C)|z| \leq\left(r\left(C_{1}\right)+|\gamma|\right)|z|=\left|C_{1} z\right|+|\gamma z| \leq\left(\left|C_{1}\right|+\left|\gamma \mathrm{I}_{\mathrm{p}}\right|\right)|z| \leq A|z| .
$$

If we assume that $r(A) \leq r(C)$, then we have

$$
r(A)|z| \leq A|z| .
$$

Next we show that this inequality can only hold true if $|z|=0$ or if $|z|$ is strictly positive and a right eigenvector of $A$ to the eigenvalue $r(A)$ (cf. (Vere-Jones, 1967. Lemma 5.2)): If $|z|=0$, then (9) holds trivially true. Hence we assume that $|z| \neq 0$. Lemma 3 implies that there exists a strictly positive left eigenvector $y \in \ell^{q}$ associated to the operator $A$. Hölders inequality and the fact that $|z| \in \ell^{p}$ imply

$$
\frac{1}{r(A)} y A|z|=y \cdot|z|=\sum_{n=1}^{\infty} x_{n} \alpha_{n}<\infty
$$

Thus we have $y \cdot(A|z|-r(A)|z|)=0$ and since $y$ is strictly positive this can only hold true if $|z|$ is an eigenvector of $A$ to the eigenvalue $r(A)$. The same way as in the proof of Lemma 3 one can now show that the irreducibility of $A_{1}$ implies the strict positivity of the eigenvector $|z|$.

It remains to show that $r(A) \leq r(C)$ yields a contradiction. Since $z$ is an eigenvector (of $C_{1}^{n}$ ) we clearly have $|z| \neq 0$. Hence, let us assume that $|z|$ is a strictly positive eigenvector of $A$. We obtain

$$
A|z|=r(A)|z| \leq r(C)|z| \leq\left(\left|C_{1}\right|+\left|\gamma \mathrm{I}_{\mathrm{p}}\right|\right)|z| \leq A|z| .
$$

Thus, we have $\left(A-\left(\left|C_{1}\right|+\left|\gamma \mathrm{I}_{\mathrm{p}}\right|\right)\right)|z|=0$. But since $|z|$ is strictly positive and $A \geq\left|C_{1}\right|+\left|\gamma \mathrm{I}_{\mathrm{p}}\right|$ but $A \neq\left|C_{1}\right|+\left|\gamma \mathrm{I}_{\mathrm{p}}\right|$, this is impossible.

Remark 3 Let $A$ be given as in Proposition 1$]$ Furthermore, let $B$ be obtained through eliminating the first row and first column of $A$, that is $B=B_{1}+\alpha \mathrm{I}_{\mathrm{p}}$, where $B_{1}=\left(\left(B_{1}\right)_{i j}\right)_{1 \leq i, j<\infty}$ is defined by $\left(B_{1}\right)_{i j}=\left(A_{1}\right)_{i+1 j+1}$. Then we have

$$
r(B)<r(A) .
$$

In order to see this, note that $B$ is also compact, $r(A)=r\left(A_{1}\right)+\alpha$ and $r(B)=r\left(B_{1}\right)+\alpha$. It is easy to show that Proposition 1 ( with $\alpha=\gamma=0$ ) implies $r\left(B_{1}\right)<r\left(A_{1}\right)$, which shows the desired result. 
Lemma 4 Let the function $\mathbf{F}$ satisfy the assumptions of Theorem 1 Then we have that the map

$$
(x, \mathbf{y}) \mapsto r\left(\frac{\partial \mathbf{F}}{\partial \mathbf{y}}(x, \mathbf{y}, \mathbf{0})\right)
$$

is continuous for all positive $(x, \mathbf{y}) \in B \times U$. Furthermore, if there exists an arbitrary point $(\tilde{x}, \tilde{\mathbf{y}}, \tilde{\mathbf{v}}) \in$ $B \times U \times V$ such that

$$
r\left(\frac{\partial \mathbf{F}}{\partial \mathbf{y}}(\tilde{x}, \tilde{\mathbf{y}}, \tilde{\mathbf{v}})\right)<1,
$$

then the same holds true in a neighborhood of $(\tilde{x}, \tilde{\mathbf{y}}, \tilde{\mathbf{v}})$.

Proof: First note, that $(x, \mathbf{y}) \mapsto \frac{\partial \mathbf{F}}{\partial \mathbf{y}}(x, \mathbf{y}, \mathbf{0})=A(x, \mathbf{y})+\alpha(x, \mathbf{y})$ is continuous. Let us fix some positive $(x, \mathbf{y}) \in B \times U$ (in the following, we suppress $x$ and $\mathbf{y}$ for brevity). The positivity properties of $\mathbf{F}$ and Lemma 3 imply that $r(\partial \mathbf{F} / \partial \mathbf{y})=r(A)+\alpha$. (Note, that we have $\sigma(\partial \mathbf{F} / \partial \mathbf{y})=\sigma(A)+\alpha$.) Furthermore, we have (compare with the proof of Lemma 3 )

$$
r(A)^{n}=r\left(A^{n}\right) .
$$

Thus it remains to show that $r\left(A^{n}\right)$ is continuous for positive $(x, \mathbf{y})$. Let $r\left(A^{n}\right)>0$. Since $A^{n}$ is compact and isolated eigenvalues with finite multiplicity must vary continuously (see (Kato, 1966, Chapter IV, $\S 3.5)$ ), we obtain the desired result. If $r\left(A^{n}\right)=0$, then the continuity follows from the upper semicontinuity of the spectrum of closed operators (see (Kato, 1966. Chapter IV, $\S 3.1$, Theorem 3.1)).

Now suppose that there exists a point $(\tilde{x}, \tilde{\mathbf{y}}, \tilde{\mathbf{v}}) \in B \times U \times V$ such that

$$
r:=r\left(\frac{\partial \mathbf{F}}{\partial \mathbf{y}}(\tilde{x}, \tilde{\mathbf{y}}, \tilde{\mathbf{v}})\right)<1 .
$$

This means, that the spectrum of $(\partial \mathbf{F} / \partial \mathbf{y})(\tilde{x}, \tilde{\mathbf{y}}, \tilde{\mathbf{v}})$ is contained in a ball with radius $r$. We can use again (Kato, 1966, Chapter IV, $\S 3.1$, Theorem 3.1) (the upper semi-continuity of the spectrum of closed operators) in order to deduce that there exists a neighborhood $D$ of $(\tilde{x}, \tilde{\mathbf{y}}, \tilde{\mathbf{v}})$ such that for all $(x, \mathbf{y}, \mathbf{v}) \in D$ the spectrum of $(\partial \mathbf{F} / \partial \mathbf{y})(x, \mathbf{y}, \mathbf{v})$ is contained in a ball with radius $1-(1-r) / 2$. In particular, it follows that

$$
r\left(\frac{\partial \mathbf{F}}{\partial \mathbf{y}}(x, \mathbf{y}, \mathbf{v})\right) \leq 1-(1-r) / 2<1
$$

This proves the second assertion of Lemma 4

\section{Proof of Theorem 1 and Corollary 1}

Proof Proof of Theorem 1; First, we fix the vector $\mathbf{v}=\mathbf{0}$. The implicit function theorem for Banach spaces (see for example (Deimling, 1985, Theorem 15.3)) implies that there exists a unique analytic solution $\mathbf{y}=\mathbf{y}(x, \mathbf{0})$ of the functional equation (4) in a neighborhood of $(0, \mathbf{0})$. It also follows from the Banach fixed-point theorem that the sequence $\mathbf{y}^{(0)} \equiv \mathbf{0}$ and

$$
\mathbf{y}^{(n+1)}(x, \mathbf{0})=\mathbf{F}\left(x, \mathbf{y}^{(n)}(x, \mathbf{0}), \mathbf{0}\right), \quad n \geq 1,
$$


converges uniformly to the unique solution $\mathbf{y}(x, \mathbf{0})$ of (4). Since $\mathbf{F}$ is positive for $\mathbf{v}=\mathbf{0}$, we get that $\mathbf{y}(x, \mathbf{0})$ is positive. Next we show that

$$
\begin{aligned}
& \mathbf{y}_{0}=\mathbf{F}\left(x_{0}, \mathbf{y}_{0}, \mathbf{0}\right), \\
& r\left(\frac{\partial \mathbf{F}}{\partial \mathbf{y}}\left(x_{0}, \mathbf{y}_{0}, \mathbf{0}\right)\right)=1,
\end{aligned}
$$

holds true. The first equation follows from analyticity. Since $\mathbf{F}$ is positive, we obtain that the Jacobian operator (evaluated at $x, \mathbf{y}(x, \mathbf{0})$ and $\mathbf{0}$ ) is positive. Lemma 4 and Proposition 1 imply that the function

$$
x \mapsto r\left(\frac{\partial \mathbf{F}}{\partial \mathbf{y}}(x, \mathbf{y}(x, \mathbf{0}), \mathbf{0})\right)
$$

is continuous and strictly monotonically increasing. We get for each $x<x_{0}$ that

$$
r\left(\frac{\partial \mathbf{F}}{\partial \mathbf{y}}(x, \mathbf{y}(x, \mathbf{0}), \mathbf{0})\right)<1 .
$$

In order to see this note that implicit differentiation yields

$$
\left(I-\frac{\partial \mathbf{F}}{\partial \mathbf{y}}(x, \mathbf{y}(x, \mathbf{0}), \mathbf{0})\right) \frac{\partial \mathbf{y}}{\partial x}(x, \mathbf{0})=\frac{\partial \mathbf{F}}{\partial x}(x, \mathbf{y}(x, \mathbf{0}), \mathbf{0}) .
$$

Suppose that the spectral radius of the (positive and irreducible) Jacobian operator at $(x, \mathbf{y}(x, \mathbf{0}), \mathbf{0})$ for some $x<x_{0}$ is equal to 1 . Lemma 3 implies that there exists a strictly positive left eigenvector to the eigenvalue 1. Multiplying this vector to equation (11) from the left yields a contradiction since $\frac{\partial \mathbf{F}}{\partial x}(x, \mathbf{y}(x, \mathbf{0}), \mathbf{0}) \neq \mathbf{0}$ (note that $\mathbf{F}(x, \mathbf{0}, \mathbf{0}) \not \equiv \mathbf{0}$ and that $\mathbf{F}$ is positive). Since $\mathbf{y}$ cannot be analytically continued at the point $x_{0}$ and since $\left(x_{0}, \mathbf{y}\left(x_{0}\right)\right)=\left(x_{0}, \mathbf{y}_{0}\right)$ lies in the domain of analyticity of $\mathbf{F}$, we obtain that 10 ) holds true. Indeed, otherwise the implicit function theorem would imply that there exists an analytic continuation.

Next, we divide equation (4) up into two equations (we project equation (4) onto the subspace spanned by the first standard vector and onto its complement):

$$
\begin{aligned}
y_{1} & =\mathbf{F}_{1}\left(x, y_{1}, \overline{\mathbf{y}}, \mathbf{0}\right), \\
\overline{\mathbf{y}} & =\overline{\mathbf{F}}\left(x, y_{1}, \overline{\mathbf{y}}, \mathbf{0}\right),
\end{aligned}
$$

where $\overline{\mathbf{y}}=S_{\ell} \mathbf{y}, \overline{\mathbf{F}}=S_{\ell} \mathbf{F}$ and $S_{\ell}$ denotes the left shift defined by $S_{\ell}\left(x_{1}, x_{2}, x_{3}, \ldots\right)=\left(x_{2}, x_{3}, \ldots\right)$. Observe, that the Jacobian operator of $\overline{\mathbf{F}}$ (with respect to $\overline{\mathbf{y}}$ ) can be obtained by deleting the first row and column of the matrix of the Jacobian operator of $\mathbf{F}$. The tuple $\left(x_{0},\left(\mathbf{y}_{0}\right)_{1}, \overline{\mathbf{y}_{0}}\right)$ is a solution of (12) and (13). We can employ the implicit function theorem and obtain that there exists a unique positive analytic solution $\overline{\mathbf{y}}=\overline{\mathbf{y}}\left(x, y_{1}, \mathbf{0}\right)$ of 13 with $\overline{\mathbf{y}}(0,0, \mathbf{0})=\overline{\mathbf{0}}$. For simplicity, we use the abbreviation $y_{01}=\left(\mathbf{y}_{0}\right)_{1}$ and $\overline{\mathbf{y}_{0}}=S_{\ell} \mathbf{y}_{0}$. Set

$$
A=\frac{\partial \mathbf{F}}{\partial \mathbf{y}}\left(x_{0}, \mathbf{y}_{0}, \mathbf{0}\right) \quad \text { and } \quad B=\frac{\partial \overline{\mathbf{F}}}{\partial \overline{\mathbf{y}}}\left(x_{0}, y_{01}, \overline{\mathbf{y}_{0}}, \mathbf{0}\right) .
$$


Proposition 1 and Remark 3 implies that $r(B)<r(A)=1$. Thus, we can employ the implicit function theorem another time (at the point $\left.\left(x_{0}, y_{01}, \overline{\mathbf{y}_{0}}, \mathbf{0}\right)\right)$ and obtain that $\overline{\mathbf{y}}\left(x, y_{1}, \mathbf{0}\right)$ is also analytic in a neighborhood of $\left(x_{0}, y_{01}, \mathbf{0}\right)$. Furthermore, we have $\overline{\mathbf{y}}\left(x_{0}, y_{01}, \mathbf{0}\right)=\overline{\mathbf{y}_{0}}$. If we insert this function into equation (12, we get a single equation

$$
y_{1}=\mathbf{F}_{1}\left(x, y_{1}, \overline{\mathbf{y}}\left(x, y_{1}, \mathbf{0}\right), \mathbf{0}\right)
$$

for $y_{1}=y_{1}(x, \mathbf{0})$. The function $G\left(x, y_{1}\right)=\mathbf{F}_{1}\left(x, y_{1}, \overline{\mathbf{y}}\left(x, y_{1}, \mathbf{0}\right), \mathbf{0}\right)$ is an analytic function around $(0,0, \mathbf{0})$ with $G\left(0, y_{1}\right)=0$ and such that all Taylor coefficients of $G$ are real and non-negative (this follows from the positivity of $\mathbf{F}$ and $\left.\mathbf{y}\left(x, y_{1}, \mathbf{0}\right)\right)$. Furthermore, the tuple $\left(x_{0}, y_{01}, \mathbf{0}\right)$ belongs to the region of convergence of $G(x, y)$. In what follows, we show that $\left(x_{0}, y_{01}, \mathbf{0}\right)$ is a positive solution of the system of equations

$$
\begin{aligned}
y_{1} & =G\left(x, y_{1}\right), \\
1 & =G_{y_{1}}\left(x, y_{1}\right),
\end{aligned}
$$

with $G_{x}\left(x_{0}, y_{01}\right) \neq 0$ and $G_{y_{1} y_{1}}\left(x_{0}, y_{01}\right) \neq 0$.

In order to see that $G_{y_{1}}\left(x_{0}, y_{01}\right)$ is indeed equal to 1 , note that the classical implicit function theorem otherwise implies that there exists an analytic solution of $y_{1}=G\left(x, y_{1}\right)$ locally around $x_{0}$. Inserting this function into equation (13), we obtain that there also exists an analytic solution $\mathbf{y}(x, \mathbf{0})$ of (4) in a neighborhood of $x_{0}$. As in $(11)$, implicit differentiation yields a contradiction since the spectral radius of the (positive and irreducible) Jacobian operator of $\mathbf{F}$ at $\left(x_{0}, \mathbf{y}_{0}, \mathbf{0}\right)$ is equal to 1.

Next suppose that $G_{x}\left(x_{0}, y_{01}\right)=0$. The positivity implies that the unique solution of $y_{1}=G\left(x, y_{1}\right)$ is given by $y_{1}(x, \mathbf{0}) \equiv 0$. Consider the solution $\mathbf{y}(x, \mathbf{0})$ of (4) for some real $x>0$ in the near of 0 . Since the spectral radius of the Jacobian operator is smaller than 1 (for $x$ small), we can express the resolvent with the aid of the Neumann series, i.e., we have (cf. [11)

$$
\begin{aligned}
\frac{\partial \mathbf{y}}{\partial x}(x, \mathbf{0}) & =\left(I-\frac{\partial \mathbf{F}}{\partial \mathbf{y}}(x, \mathbf{y}(x), \mathbf{0})\right)^{-1} \frac{\partial \mathbf{F}}{\partial x}(x, \mathbf{y}(x), \mathbf{0}) \\
& =\sum_{n \geq 0}\left(\frac{\partial \mathbf{F}}{\partial \mathbf{y}}(x, \mathbf{y}(x), \mathbf{0})\right)^{n} \frac{\partial \mathbf{F}}{\partial x}(x, \mathbf{y}(x), \mathbf{0})
\end{aligned}
$$

Since $\partial \mathbf{F} / \partial \mathbf{y}$ is irreducible and $\partial \mathbf{F} / \partial x \neq 0$ we obtain that no component of the solution $\mathbf{y}(x, \mathbf{0})$ is a constant function. In particular, $y_{1}(x, \mathbf{0})$ cannot be constant.

Finally, if $G_{y_{1} y_{1}}\left(x_{0}, y_{01}\right)=0$, it follows from the positivity of $G$ that $G$ is a linear function in $y_{1}$. But then the conditions $G\left(x_{0}, y_{01}\right)=y_{01}$ and $G_{y_{1}}\left(x_{0}, y_{01}\right)=1$ imply

$$
y_{01}=G\left(x, y_{01}\right)=G(x, 0)+G_{y_{1}}\left(x, y_{01}\right) \cdot y_{01}=G(x, 0)+y_{01}
$$

Thus we have in this case that $G(x, 0)=0$. But then ( since $G_{y_{1}}\left(x, y_{1}\right)=G(x, 1)$ and $\left.G\left(0, y_{1}\right)=0\right)$, the only solution of

$$
y_{1}=G\left(x, y_{1}\right)=G_{y_{1}}\left(x, y_{01}\right) \cdot y_{1}
$$

is $y_{1}(x, \mathbf{0}) \equiv 0$. As we have seen before, this is impossible. 
It follows from (Drmota 2009, Theorem 2.19) that there exists a unique solution $\tilde{y}\left(x_{1}, \mathbf{0}\right)$ of the equation $y_{1}=G\left(x, y_{1}\right)$ with $\tilde{y}_{1}(0, \mathbf{0})=0$. It is analytic for $|x|<x_{0}$ and there exist functions $g_{1}(x, \mathbf{0})$ and $h_{1}(x, \mathbf{0})$ that are analytic around $x_{0}$ such that $\tilde{y}_{1}(x, \mathbf{0})$ has a representation of the form

$$
\tilde{y}_{1}(x, \mathbf{0})=g_{1}(x, \mathbf{0})-h_{1}(x, \mathbf{0}) \sqrt{1-\frac{x}{x_{0}}}
$$

locally around $x_{0}$ with $h_{1}\left(x_{0}, \mathbf{0}\right)>0$ and $g_{1}\left(x_{0}, \mathbf{0}\right)=y_{01}$. Due to the uniqueness of the solution $y(x, \mathbf{0})$ of the functional equation (4), we have that the first component of $y(x, \mathbf{0})$ coincide with $\tilde{y}_{1}(x, \mathbf{0})$, i.e., $y_{1}(x, \mathbf{0})=\tilde{y}_{1}(x, \mathbf{0})$. Moreover, we get $\overline{\mathbf{y}}\left(x, y_{1}(x, \mathbf{0}), \mathbf{0}\right)=\left(y_{2}(x, \mathbf{0}), y_{3}(x, \mathbf{0}), \ldots\right)$. More precisely, the analyticity of $\overline{\mathbf{y}}$ implies that there exist an $s>0$ and vectors $a_{n}(x):=a_{n}\left(x, g_{1}(x, \mathbf{0}), \mathbf{0}\right) \in \ell^{p}$ such that $\sum_{n}\left\|a_{n}(x)\right\| s^{n}<\infty$ and

$$
\overline{\mathbf{y}}\left(x, y_{1}, \mathbf{0}\right)=\overline{\mathbf{y}}\left(x, g_{1}(x, \mathbf{0}), \mathbf{0}\right)+\sum_{n \geq 1} \frac{a_{n}(x)}{n !}\left(\left(y_{1}-g_{1}(x, \mathbf{0})\right)^{n}\right),
$$

and we obtain

$$
\begin{aligned}
\overline{\mathbf{y}}\left(x, y_{1}(x, \mathbf{0}), \mathbf{0}\right)=\overline{\mathbf{y}}\left(x, g_{1}(x, \mathbf{0}), \mathbf{0}\right)+\sum_{n \geq 1} \frac{\left(1-\frac{x}{x_{0}}\right)^{n}}{(2 n) !} a_{2 n}(x)\left(h_{1}(x, \mathbf{0})^{2 n}\right) \\
\quad-\sqrt{1-\frac{x}{x_{0}}} \sum_{n \geq 0} \frac{\left(1-\frac{x}{x_{0}}\right)^{n}}{(2 n+1) !} a_{2 n+1}(x)\left(h_{1}(x, \mathbf{0})^{2 n+1}\right) \\
=\overline{\mathbf{g}}(x, \mathbf{0})-\overline{\mathbf{h}}(x, \mathbf{0}) \sqrt{1-\frac{x}{x_{0}}}
\end{aligned}
$$

In particular, we get the desired representation

$$
\mathbf{y}(x, \mathbf{0})=\mathbf{g}(x, \mathbf{0})-\mathbf{h}(x, \mathbf{0}) \sqrt{1-\frac{x}{x_{0}}}
$$

with $\mathbf{g}(x, \mathbf{0})=\left(g_{1}(x, \mathbf{0}), \overline{\mathbf{g}}(x, \mathbf{0})\right)$ and $\mathbf{h}(x, \mathbf{0})=\left(h_{1}(x, \mathbf{0}), \overline{\mathbf{h}}(x, \mathbf{0})\right)$. Furthermore, we have the property $\mathbf{h}_{1}(x, \mathbf{0})>0$. Since the same result can be obtained when equation (4) is projected onto the subspace spanned by the $i$-th standard vector and onto its complement, we obtain that $\mathbf{h}_{i}(x, \mathbf{0})>0$, either. (Note, that the reasoning of Remark 3 also works when the $i$-th row and column of the Jacobian matrix is deleted.)

Until now, we have shown that the statement of Theorem 1 is true for $\mathbf{v}=\mathbf{0}$. Next, we prove that the solution $\mathbf{y}(x, \mathbf{v})$ is also analytic in $\mathbf{v}$. We have seen before, that

$$
r\left(\frac{\partial \bar{F}}{\partial \overline{\mathbf{y}}}\left(x_{0},\left(\mathbf{y}_{0}\right)_{1}, \overline{\mathbf{y}_{0}}, \mathbf{0}\right)\right)<1 .
$$

It follows, that there exists a unique solution $\overline{\mathbf{y}}\left(x, y_{1}, \mathbf{v}\right)$ of the function equation

$$
\overline{\mathbf{y}}=\overline{\mathbf{F}}\left(x, y_{1}, \overline{\mathbf{y}}, \mathbf{v}\right)
$$


for all $\left(x, \mathbf{y}_{1}, \mathbf{v}\right)$ in a neighborhood of $\left(x_{0},\left(\mathbf{y}_{0}\right)_{1}, \mathbf{0}\right)$. Inserting this solution into (12) (but this time with the additional variable $\mathbf{v})$, we have already seen that the functional equations

$$
\begin{aligned}
y_{1} & =G\left(x, y_{1}, \mathbf{v}\right), \\
1 & =G_{y_{1}}\left(x, y_{1}, \mathbf{v}\right),
\end{aligned}
$$

with $G\left(x, y_{1}, \mathbf{v}\right)=\mathbf{F}_{1}\left(x, y_{1}, \overline{\mathbf{y}}\left(x, y_{1}, \mathbf{v}\right), \mathbf{v}\right)$ have a positive solution $\left(x_{0},\left(\mathbf{y}_{0}\right)_{1}, \mathbf{0}\right)$. Furthermore, note that $G_{x}\left(x_{0},\left(\mathbf{y}_{0}\right)_{1}, \mathbf{0}\right) \neq 0$ and $G_{y_{1} y_{1}}\left(x_{0},\left(\mathbf{y}_{0}\right)_{1}, \mathbf{0}\right) \neq 0$. Since we have (evaluated at $\left.\left(x_{0},\left(\mathbf{y}_{0}\right)_{1}, \mathbf{0}\right)\right)$ that

$$
\operatorname{det}\left(\begin{array}{cc}
-G_{x} & 1-G_{y_{1}} \\
-G_{y_{1}, x} & -G_{y_{1}, y_{1}}
\end{array}\right)=G_{x} \cdot G_{y_{1} y_{1}} \neq 0
$$

the implicit function theorem implies that there exist unique analytic functions $x_{0}(\mathbf{v})$ and $y_{1}(\mathbf{v})$ in a neighborhood of $\mathbf{0}$, such that we have $y_{1}(\mathbf{v})=G\left(x_{0}(\mathbf{v}), y_{1}(\mathbf{v}), \mathbf{v}\right)$ and $G_{y_{1}}\left(x_{0}(\mathbf{v}), y_{1}(\mathbf{v}), \mathbf{v}\right)=1$. In particular, we have $x_{0}(\mathbf{0})=x_{0}$ and $y_{1}(\mathbf{0})=\left(\mathbf{y}_{0}\right)_{1}$. From continuity it follows that for any $\mathbf{v}$ in a neighborhood of $\mathbf{0}$ we have $G_{x}\left(x_{0}(\mathbf{v}), y_{1}(\mathbf{v}), \mathbf{v}\right) \neq 0$ and $G_{y_{1} y_{1}}\left(x_{0}(\mathbf{v}), y_{1}(\mathbf{v}), \mathbf{v}\right) \neq 0$. Thus, the Weierstrass preparation theorem implies that there exist analytic functions $g_{1}(x, \mathbf{v})$ and $h_{1}(x, \mathbf{v})$ such that

$$
y_{1}(x, \mathbf{v})=g_{1}(x, \mathbf{v})-h_{1}(x, \mathbf{v}) \sqrt{1-\frac{x}{x_{0}(\mathbf{v})}}
$$

(see for example the proof of (Drmota, 2009, Theorem 2.19)). Inserting this solution into $\overline{\mathbf{y}}\left(x, y_{1}, \mathbf{v}\right)$ (cf. 15), this finally proves (5).

In what follows, we show that $x_{0}(\mathbf{v})$ is the only singularity of $\mathbf{y}(x, \mathbf{v})$ on the circle $|x|=x_{0}(\mathbf{v})$. Recall that by assumption, there exist two integers $n_{1}$ and $n_{2}$ that are relatively prime such that $\left[x^{n_{1}}\right] \mathbf{y}_{1}(x, \mathbf{0})>0$ and $\left[x^{n_{2}}\right] \mathbf{y}_{1}(x, \mathbf{0})>0$. In order to show the desired result, it suffices to show that

$$
G_{y_{1}}\left(x, \mathbf{y}_{1}(x, \mathbf{v}), \mathbf{v}\right) \neq 1
$$

for $|x|=x_{0}(\mathbf{v})$ but $x \neq x_{0}(\mathbf{v})$ (compare with the proof of (Drmota, 2009, Theorem 2.19)). Let us first study the case $\mathbf{v}=\mathbf{0}$. Since $\mathbf{y}_{1}(x, \mathbf{0})$ is positive, we clearly have $\left|\mathbf{y}_{1}(x, \mathbf{0})\right| \leq \mathbf{y}_{1}(|x|, \mathbf{0})$. If equality occurs, then

$$
x^{n_{1}}=|x|^{n_{1}}=x_{0}^{n_{1}} \quad \text { and } \quad x^{n_{2}}=|x|^{n_{2}}=x_{0}^{n_{2}} .
$$

Since $n_{1}$ and $n_{2}$ are relatively prime we obtain $x=x_{0}$, which is impossible. Thus, we actually have $\left|\mathbf{y}_{1}(x, \mathbf{0})\right|<\mathbf{y}_{1}(|x|, \mathbf{0})$. The positivity of $G$ implies

$$
\begin{aligned}
\left|G_{y_{1}}\left(x, \mathbf{y}_{1}(x, \mathbf{0}), \mathbf{0}\right)\right| & \leq G_{y_{1}}\left(|x|,\left|\mathbf{y}_{1}(x, \mathbf{0})\right|, \mathbf{0}\right) \\
& <G_{y_{1}}\left(|x|, \mathbf{y}_{1}(|x|, \mathbf{0}), \mathbf{0}\right)=G_{y_{1}}\left(x_{0},\left(\mathbf{y}_{0}\right)_{1}, \mathbf{0}\right)=1 .
\end{aligned}
$$

From continuity we obtain that $\left|G_{y_{1}}\left(x, \mathbf{y}_{1}(x, \mathbf{v}), \mathbf{v}\right)\right|<1$ and 17 follows. Thus, there exists an analytic continuation of $\mathbf{y}_{1}(x, \mathbf{v})$ locally around $x$. From positivity, it follows that

$$
\left|\frac{\partial \overline{\mathbf{F}}}{\partial \overline{\mathbf{y}}}\left(x,(\mathbf{y}(x, \mathbf{0}))_{1}, \overline{\mathbf{y}}(x, \mathbf{0}), \mathbf{0}\right)\right| \leq\left|\frac{\partial \overline{\mathbf{F}}}{\partial \overline{\mathbf{y}}}\left(x_{0}, y_{01}, \overline{\mathbf{y}}_{0}, \mathbf{0}\right)\right| .
$$


Employing Proposition 1 yields

$$
r\left(\frac{\partial \overline{\mathbf{F}}}{\partial \overline{\mathbf{y}}}\left(x^{\prime}, \mathbf{y}\left(x^{\prime}, \mathbf{v}\right), \mathbf{v}\right)\right)<1
$$

for $x^{\prime}=x$ and $\mathbf{v}=\mathbf{0}$. Lemma 4 implies that the same holds true for all $\left(x^{\prime}, \mathbf{v}\right)$ in a neighborhood of $(x, \mathbf{0})$. The implicit function theorem implies that we can insert the function $\mathbf{y}_{1}(x, \mathbf{v})$ into the solution of equation (13). We obtain that $x_{0}(\mathbf{v})$ is the only singularity of $\mathbf{y}(x, \mathbf{v})$ on the circle $|x|=x_{0}(\mathbf{v})$ and there exist constants $\delta$ and $\eta$ such that $\mathbf{y}(x, \mathbf{v})$ is analytic in $\left\{x:|x|<x_{0}(\mathbf{v})+\eta, \mid \arg \left(x / x_{0}(\mathbf{v})-1\right)>\delta\right\}$ (note, that locally around $x_{0}(\mathbf{v})$ the representation (16) yields an analytic continuation).

Proof Proof of Corollary $\mathbf{1}$; The first part of the proof is similar to (15). Since $G(x, \mathbf{y}, \mathbf{v})$ is analytic in $\left(x_{0}(\mathbf{0}), \mathbf{y}\left(x_{0}(\mathbf{0}), \mathbf{0}\right), \mathbf{0}\right)$ there exist an $s>0$ and continuous symmetric $n$-linear forms $A_{n}(x, \mathbf{v}):=$ $A_{n}(x, \mathbf{g}(x, \mathbf{v}), \mathbf{v})$ (defined on the the right space) such that

$$
\sum_{n \geq 1}\left\|A_{n}(x, \mathbf{v})\right\| s^{n}<\infty
$$

and

$$
G(x, \mathbf{y}, \mathbf{v})=G(x, \mathbf{g}(x, \mathbf{v}), \mathbf{v})+\sum_{n \geq 1} \frac{A_{n}(x, \mathbf{v})}{n !}\left((\mathbf{y}-\mathbf{g}(x, \mathbf{v}))^{n}\right)
$$

Note, that

$$
A_{1}(x, \mathbf{v})(\mathbf{y})=\frac{\partial G}{\partial \mathbf{y}}(x, \mathbf{g}(x, \mathbf{v}), \mathbf{v}) \in \ell^{q}
$$

and

$$
A_{1}\left(x_{0}(\mathbf{0}), \mathbf{0}\right)=\frac{\partial G}{\partial \mathbf{y}}\left(x_{0}(\mathbf{0}), \mathbf{y}\left(x_{0}(\mathbf{0}), \mathbf{0}\right), \mathbf{0}\right) \neq \mathbf{0}
$$

by assumption. We can write

$$
\begin{aligned}
G(x, \mathbf{y}(x, \mathbf{v}), \mathbf{v})= & G(x, \mathbf{g}(x, \mathbf{v}), \mathbf{v})+\sum_{n \geq 1} \frac{\left(1-\frac{x}{x_{0}(\mathbf{v})}\right)^{n}}{(2 n) !} A_{2 n}(x, \mathbf{v})\left(\mathbf{h}(x, \mathbf{v})^{2 n}\right) \\
& \quad-\sqrt{1-\frac{x}{x_{0}(\mathbf{v})}} \sum_{n \geq 0} \frac{\left(1-\frac{x}{x_{0}(\mathbf{v})}\right)^{n}}{(2 n+1) !} A_{2 n+1}(x, \mathbf{v})\left(\mathbf{h}(x, \mathbf{v})^{2 n+1}\right) \\
= & \bar{g}(x, \mathbf{v})-\bar{h}(x, \mathbf{v}) \sqrt{1-\frac{x}{x_{0}(\mathbf{v})}}
\end{aligned}
$$

Moreover, we have that $\bar{g}$ and $\bar{h}$ are analytic and

$$
\bar{h}\left(x_{0}(\mathbf{0}), \mathbf{0}\right)=A_{1}\left(x_{0}(\mathbf{0}), \mathbf{0}\right) \cdot \mathbf{h}\left(x_{0}(\mathbf{0}), \mathbf{0}\right) \neq 0 .
$$

(Recall that $\mathbf{h} \in \ell^{p}$ and $\mathbf{h}_{i}\left(x_{0}(\mathbf{0}), \mathbf{0}\right)>0$ for all $i \geq 1$, see Theorem 11). The analyticity of $x_{0}(\mathbf{v})$ and $G(x, \mathbf{y}(x, \mathbf{v}), \mathbf{v})$ follows from Theorem 1 . Using the transfer lemma of Flajolet and Odlyzko (1990) (the region of analyticity $\Delta$ from Theorem 1 is uniform in $\mathbf{v}$ ) we finally obtain that

$$
\left[x^{n}\right] G(x, \mathbf{y}(x, \mathbf{v}), \mathbf{v})=\frac{\bar{h}\left(x_{0}(\mathbf{v}), \mathbf{v}\right)}{2 \sqrt{\pi}} x_{0}(\mathbf{v})^{-n} n^{-3 / 2}\left(1+O\left(\frac{1}{n}\right)\right)
$$


uniformly for $\mathbf{v}$ in a neighborhood of $\mathbf{0}$. (Note, that the part coming from $g(x, \mathbf{v})$ is exponentially smaller than the stated term.)

\section{Proof of Theorem 2 and Corollary 2}

Recall that $G(x, \mathbf{y}, \mathbf{v})$ is the generating function of some combinatorial object of the form

$$
G(x, \mathbf{y}(x, \mathbf{v}), \mathbf{v})=\sum_{n=0}^{\infty} c_{n}(\mathbf{v}) x^{n}
$$

where $\mathbf{y}(x, \mathbf{v})$ satisfies a functional equation

$$
\mathbf{y}=\mathbf{F}(x, \mathbf{y}, \mathbf{v})
$$

with $\mathbf{y}(0, \mathbf{v})=\mathbf{0}$ such that the assumptions of Theorem 1 are satisfied. Moreover, $\mathbf{X}_{n}$ denotes an $\ell^{p}$ valued $(1 \leq p<\infty)$ random variable defined on some probability space $(\Omega, \mathcal{F}, \mathbb{P})$ with

$$
\mathbb{E}\left[e^{i t \boldsymbol{\ell} \cdot \mathbf{X}_{n}}\right]=\frac{c_{n}(i t \boldsymbol{\ell})}{c_{n}(\mathbf{0})}
$$

for all $\ell \in \ell^{q}$.

Proof of Theorem 2; We have $\ell \in \ell^{q}$. Corollary 1 implies that uniformly in $t$ (for small values of $t$ ) that

$$
c_{n}(i t \ell)=\frac{\bar{h}\left(x_{0}(i t \ell), i t \ell\right)}{2 \sqrt{\pi}} x_{0}(i t \ell)^{-n} n^{-3 / 2}\left(1+O\left(\frac{1}{n}\right)\right)
$$

where $\bar{h}$ and $x_{0}$ are analytic functions. Thus we get

$$
\mathbb{E}\left[e^{i t \boldsymbol{\ell} \cdot \mathbf{X}_{n}}\right]=\frac{c_{n}(i t \boldsymbol{\ell})}{c_{n}(\mathbf{0})}=\frac{\bar{h}\left(x_{0}(i t \boldsymbol{\ell}), i t \boldsymbol{\ell}\right)}{\bar{h}\left(x_{0}(\mathbf{0}), \mathbf{0}\right)}\left(\frac{x_{0}(\mathbf{0})}{x_{0}(i t \ell)}\right)^{n}\left(1+O\left(\frac{1}{n}\right)\right) .
$$

Set

$$
\Phi_{\ell}(s):=x_{0}(s \boldsymbol{\ell}), \quad f_{\boldsymbol{\ell}}(s)=\log \left(\frac{\Phi_{\boldsymbol{\ell}}(0)}{\Phi_{\boldsymbol{\ell}}(s)}\right), \quad \text { and } \quad g_{\boldsymbol{\ell}}(s)=\log \left(\frac{\bar{h}\left(\Phi_{\boldsymbol{\ell}}(s), s \boldsymbol{\ell}\right)}{\bar{h}\left(\Phi_{\boldsymbol{\ell}}(0), \mathbf{0}\right)}\right) .
$$

These functions are analytic in a neighborhood of $s=0$ and we have $f_{\ell}(0)=g_{\ell}(0)=0$ and $\Phi_{\ell}(0)=$ $x_{0}(\mathbf{0})$. We obtain

$$
\begin{aligned}
\mathbb{E}\left[e^{i t \boldsymbol{\ell} \cdot \mathbf{X}_{n}}\right] & =e^{f_{\ell}(i t) \cdot n+g_{\ell}(i t)}\left(1+O\left(\frac{1}{n}\right)\right) \\
& =e^{i t \mu_{\ell} n-\sigma_{\ell}^{2} n \frac{t^{2}}{2}+O\left(n t^{3}\right)+O(t)}\left(1+O\left(\frac{1}{n}\right)\right)
\end{aligned}
$$

where $\mu_{\ell}=f_{\ell}^{\prime}(0)$ and $\sigma_{\ell}^{2}=f_{\ell}^{\prime \prime}(0)$. Replacing $t$ by $t / \sqrt{n}$ we get

$$
\mathbb{E}\left[e^{i t \boldsymbol{\ell} \cdot \mathbf{X}_{n} / \sqrt{n}}\right]=e^{i t \mu_{\ell} \sqrt{n}-\sigma_{\ell}^{2} \frac{t^{2}}{2}+O(t / \sqrt{n})}\left(1+O\left(\frac{1}{n}\right)\right) .
$$


By definition, $\boldsymbol{\ell} \cdot \mathbb{E} \mathbf{X}_{n}=\mathbb{E}\left[\boldsymbol{\ell} \cdot \mathbf{X}_{n}\right]$. If we set $\chi_{n}(t)=\mathbb{E} e^{i t \boldsymbol{\ell} \cdot \mathbf{X}_{n}}$, then $\mathbb{E}\left[\boldsymbol{\ell} \cdot \mathbf{X}_{n}\right]=-i \cdot \chi_{n}^{\prime}(0)$. By Cauchy's formula, we have

$$
-i \cdot \chi_{n}^{\prime}(0)=-\frac{1}{2 \pi} \int_{|u|=\rho} \frac{\chi_{n}(u)}{u^{2}} \mathrm{~d} u
$$

Setting $\rho=1 / n$, we get

$$
\begin{aligned}
\mathbb{E}\left[\boldsymbol{\ell} \cdot \mathbf{X}_{n}\right] & =-\frac{1}{2 \pi} \int_{|u|=1 / n} \frac{1+i u \mu_{\ell} n+i u g_{\ell}^{\prime}(0)+O\left(u^{2}\right)}{u^{2}}\left(1+O\left(\frac{1}{n}\right)\right) \mathrm{d} u \\
& =\frac{1}{2 \pi i} \int_{|u|=1 / n} \frac{\mu_{\ell} n}{u} \mathrm{~d} u+O(1) .
\end{aligned}
$$

This implies $\boldsymbol{\ell} \cdot \mathbb{E} \mathbf{X}_{n}=\mu_{\ell} n+O(1)$. Setting

$$
\mathbf{Y}_{n}:=\frac{\mathbf{X}_{n}-\mathbb{E} \mathbf{X}_{n}}{\sqrt{n}}
$$

we finally obtain (see (18))

$$
\lim _{n \rightarrow \infty} \mathbb{E}\left[e^{i t \ell \cdot \mathbf{Y}_{n}}\right]=e^{-\frac{\sigma_{\ell}^{2} t^{2}}{2}}
$$

In particular, this implies that $\mathbf{Y}_{n}$ weakly converges to a centered Gaussian random variable with variance $\sigma_{\ell}^{2}$. It remains to calculate $\mu_{\ell}$ and $\sigma_{\ell}^{2}$ : Since $x_{0}: \ell^{q} \rightarrow \mathbb{C}$ is an analytic function, it follows that there exists a vector $\partial x_{0} / \partial \mathbf{v}: \ell^{q} \rightarrow \ell^{p} \approx\left(\ell^{q}\right)^{*}$ (the first derivative) and an operator $\partial^{2} x_{0} / \partial \mathbf{v}^{2}: \ell^{q} \rightarrow L\left(\ell^{q}, \ell^{p}\right)$ (the second derivative) such that

$$
x_{0}(\mathbf{h})=x_{0}(\mathbf{0})+\frac{\partial x_{0}}{\partial \mathbf{v}}(\mathbf{0}) \cdot \mathbf{h}+\frac{1}{2}\left(\frac{\partial^{2} x_{0}}{\partial \mathbf{v}^{2}}(\mathbf{0})\right)(\mathbf{h}) \cdot \mathbf{h}+o\left(\|\mathbf{h}\|^{2}\right)
$$

in a neighborhood of $\mathbf{0}$. Note, that the second derivative can be associated with the infinite dimensional Hessian matrix $A=\left(a_{i j}\right)_{1 \leq i, j<\infty}$ via

$$
\left(\frac{\partial^{2} x_{0}}{\partial \mathbf{v}^{2}}(\mathbf{0})\right)(\mathbf{h}) \cdot \mathbf{h}=\mathbf{h}^{H} A \mathbf{h}
$$

where

$$
a_{i j}=\frac{\partial^{2} x_{0}}{\partial \mathbf{v}_{i} \mathbf{v}_{j}}(\mathbf{0})
$$

We obtain

$$
\mu_{\ell}=-\frac{1}{\Phi_{\ell}(0)} \Phi_{\ell}^{\prime}(0)=-\frac{1}{\Phi_{\ell}(0)} \cdot \frac{\partial x_{0}}{\partial \mathbf{v}}(\mathbf{0}) \cdot \boldsymbol{\ell}
$$

and

$$
\sigma_{\ell}^{2}=\frac{\Phi_{\ell}^{\prime}(0)^{2}}{\Phi_{\ell}(0)^{2}}-\frac{\Phi_{\ell}^{\prime \prime}(0)}{\Phi_{\ell}(0)}=\mu_{\ell}^{2}-\frac{\Phi_{\ell}^{\prime \prime}(0)}{\Phi_{\ell}(0)}=\mu_{\ell}^{2}-\frac{1}{\Phi_{\ell}(0)}\left(\frac{\partial^{2} x_{0}}{\partial \mathbf{v}^{2}}(\mathbf{0})\right)(\ell) \cdot \boldsymbol{\ell}
$$


If we define $B \in L\left(\ell^{q}, \ell^{p}\right)$ by

$$
\frac{1}{\Phi_{\ell}(0)^{2}}\left(\frac{\partial x_{0}}{\partial \mathbf{v}}(0) \cdot \frac{\partial x_{0}}{\partial \mathbf{v}}(0)^{T}\right)-\frac{1}{\Phi_{\ell}(0)} A
$$

then we have

$$
\sigma_{\ell}^{2}=\ell^{H} B \ell
$$

This finally proves Theorem 2

Proof of Corollary 2; Set $\mathbf{Y}_{n}=\left(\mathbf{X}_{n}-\mathbb{E} \mathbf{X}_{n}\right) / \sqrt{n}$. Since the set of laws of $\left(\mathbf{Y}_{n}\right)_{n \geq 1}$ is tight, we know from Prohorov's theorem (see (Billingsley, 1999. Chapter I, Section 5)) that the set of laws of $\left(\mathbf{Y}_{n}\right)_{n \geq 1}$ is a relatively compact set. In particular, it follows that there exists a subsequence $\left(\mathbf{Y}_{n_{k}}\right)_{k \geq 1}$ that weakly converges to some random variable $\mathbf{X}$. Let $\chi_{\boldsymbol{\ell}}(t)$ be the characteristic function of $\boldsymbol{\ell} \cdot \mathbf{X}$, that is, $\chi_{\ell}(t)=\mathbb{E} e^{i t \ell \cdot \mathbf{X}}$. From weak convergence of $\mathbf{Y}_{n_{k}}$, we obtain on the one hand that

$$
\lim _{k \rightarrow \infty} \mathbb{E}\left[e^{i t \ell \cdot \mathbf{Y}_{n_{k}}}\right]=\chi_{\ell}(t)
$$

for all $\ell \in \ell^{q}$. One the other hand, Theorem 2 implies that there exist constants $\sigma_{\ell}^{2}$ such that

$$
\lim _{n \rightarrow \infty} \mathbb{E}\left[e^{i t \ell \cdot \mathbf{Y}_{n}}\right]=e^{-\frac{\sigma_{R}^{2} t^{2}}{2}}
$$

for all $\ell \in \ell^{q}$. Hence we see that we actually have

$$
\chi_{\ell}(t)=e^{-\frac{\sigma_{\ell}^{2} t^{2}}{2}}
$$

and thus, $\mathbf{X}$ is a centered Gaussian random variable. Moreover, we obtain that not only $\left(\mathbf{Y}_{n_{k}}\right)_{k \geq 1}$ but $\left(\mathbf{Y}_{n}\right)_{n \geq 1}$ weakly converges to $\mathbf{X}$. Since the distribution of $\mathbf{X}$ is uniquely determined by the distributions of $\boldsymbol{\ell} \cdot \mathbf{X}, \boldsymbol{\ell} \in \ell^{q}$, we obtain that $\mathbf{X}$ is uniquely determined by the operator $B$ stated in Theorem 2 .

\section{Proof of Theorem 3}

For the sake of brevity we just give an outline of the proof. Corollary 2 implies that we only have to show the tightness condition. Theorem 6.2.3 of Grenander (1963) states that tightness follows from the property

$$
\lim _{N \rightarrow \infty} \sup _{n \geq 1} \mathbb{E}\left[\sum_{j>N} \frac{\left(X_{n}^{(j)}-\mathbb{E} X_{n}^{(j)}\right)^{2}}{n}\right]=0 .
$$

First of all we know by assumption that $X_{n}^{(j)}=0$ if $j>c n$. Hence the condition 19 reduces to

$$
\lim _{N \rightarrow \infty} \sup _{n \geq 1} \sum_{N<j \leq c n} \sigma_{n, j}^{2}=0
$$

where $\sigma_{n, j}^{2}$ denote the variance of the normalized random variable $\left(X_{n}^{(j)}-\mathbb{E} X_{n}^{(j)}\right) / \sqrt{n}$. 
Now assume that we know that $\sigma_{n, j}^{2}$ is asymptotically given by

$$
\sigma_{n, j}^{2}=\sigma_{j}^{2}+\frac{\tau_{j}}{n}+O\left(n^{-2}\right)
$$

where

$$
\sum_{j \geq 0} \sigma_{j}^{2}<\infty, \quad \text { and } \quad \tau_{j}=o(1) \quad(j \rightarrow \infty),
$$

and the error term is uniform for all $j \geq 0$. Then it is clear that 21 implies (20) and, hence, tightness follows.

By Theorem 2.23 of Drmota (2009) we already know that we have an expansion of the form (21) and that $\sigma_{j}^{2}$ is given by

$$
\begin{aligned}
\sigma_{j}^{2}=\frac{F_{v_{j}}}{x_{0} F_{x}} & +\left(\frac{F_{v_{j}}}{x_{0} F_{x}}\right)^{2}+\frac{1}{x_{0} F_{x}^{3} F_{y y}}\left(F_{x}^{2}\left(F_{y y} F_{v_{j} v_{j}}-F_{y v_{j}}^{2}\right)-2 F_{x} F_{v_{j}}\left(F_{y y} F_{x v_{j}}-F_{y x} F_{y v_{j}}\right)\right. \\
& \left.+F_{v_{j}}^{2}\left(F_{y y} F_{x x}-F_{y x}^{2}\right)\right)
\end{aligned}
$$

By assumption it is then clear that the sum $\sum \sigma_{j}^{2}$ is convergent. In a similar (but slightly more involved) way it is also possible to calculate $\tau_{j}$ explicitly, from which we easily deduce the convergence $\tau_{j} \rightarrow 0$.

\section{Proof of Theorem 4}

In this section we prove Theorem 4 . Let us recall that $\mathbf{F}$ can be written as

$$
F(x, \mathbf{y}, \mathbf{v})=\mathbf{L}(x, \mathbf{v}) \mathbf{y}+\mathbf{b}(x, \mathbf{v})
$$

where $\mathbf{L}(x, \mathbf{0})=A(x)+\alpha(x) \mathrm{I}_{\mathrm{p}}, \alpha$ is an analytic function, and there exists an integer $n$ such that $A^{n}$ is compact. Furthermore, $A(x)$ is irreducible and strictly positive for $x>0$ and $\alpha$ and $\mathbf{b}(x, \mathbf{0})$ has positive Taylor coefficients. Moreover, $\mathbf{F}(0, \mathbf{y}, \mathbf{v})=\mathbf{0}$ for all $\mathbf{y}$ and $\mathbf{v}$ in a neighborhood of $\mathbf{0}$.

In order to show the desired result we proceed as in the proof of Theorem 1 Let us first assume that $\mathbf{v}=\mathbf{0}$ (in the following, we suppress the variable $\mathbf{v}=\mathbf{0}$ in order to make text more readable). The implicit function theorem (and the Banach fixed-point theorem) implies that there exists a unique analytic and positive solution $\mathbf{y}(x)$ of the function equation $\mathbf{y}=\mathbf{F}(x, \mathbf{y}), \mathbf{y}(0)=\mathbf{0}$. Since $\mathbf{F}$ is linear, this can be also deduced from the following reasoning: Since $r(\mathbf{L}(0))=0$ (note, that $\mathbf{F}(0, \mathbf{y})=\mathbf{0}$ for all $\mathbf{y}$ in a neighborhood of $\mathbf{0})$, we see that the solution $\mathbf{y}(x)$ is given by

$$
\mathbf{y}(x)=\left(\mathrm{I}_{\mathrm{p}}-\mathbf{L}(x)\right)^{-1} \mathbf{b}(x)=\sum_{k=0}^{\infty} \mathbf{L}(x)^{k} \mathbf{b}(x) .
$$

Here we also used that the inverse of $\left(\mathrm{I}_{\mathrm{p}}-\mathbf{L}(x)\right)$ can be represented by the corresponding Neumann series as long as $r(\mathbf{L}(x))<1$. Since $\mathbf{L}$ and $\mathbf{b}$ is positive, the solution $\mathbf{y}$ is also positive. Note, that the solution exists for all $x<x_{0}$ and that there is a singularity at $x_{0}$.

In what follows we split the functional equation $\mathbf{y}=\mathbf{F}(x, \mathbf{y})$ up into two equations (cf. (12) and (13)). Since $\mathbf{F}$ is linear, this gives

$$
\begin{aligned}
y_{1} & =\mathbf{l}_{11}(x) \cdot \mathbf{y}_{1}+\overline{\mathbf{l}_{r}}(x) \cdot \overline{\mathbf{y}}+\mathbf{b}_{1}(x) \\
\overline{\mathbf{y}} & =\mathbf{y}_{1} \overline{\mathbf{l}_{c}}(x)+\overline{\mathbf{L}}(x) \overline{\mathbf{y}}+\overline{\mathbf{b}}(x)
\end{aligned}
$$


where we denote by $\mathbf{l}_{r}$ the vector in $\ell^{p}$ associated to the first row of the infinite matrix $\mathbf{L}$ (that is, $\mathbf{l}_{r}^{T}=$ $e_{1}^{T} \mathbf{L}$ ), by $\mathbf{l}_{c}$ the first column of $\mathbf{L}$, and by $\mathbf{l}_{11}$ the element $e_{1}^{T} \mathbf{L} e_{1}$. The operator $\overline{\mathbf{L}}$ is defined as the operator $S_{\ell} \mathbf{L} S_{r}$, where $S_{\ell}$ is the left shift- and $S_{r}$ is the right shift operator; moreover we set $\overline{\mathbf{a}}=S_{\ell} \mathbf{a}$. Note that the matrix representation of $\overline{\mathbf{L}}$ is equal to the matrix representation of $\mathbf{L}$ without the first row and column.

Since $r\left(\mathbf{L}\left(x_{0}\right)\right)=1$, we obtain (cf. Remark 3 ) that $r\left(\mathbf{L}\left(x_{0}\right)\right)<1$. Thus, the solution of 23 is given by

$$
\overline{\mathbf{y}}\left(x, \mathbf{y}_{1}\right)=\left(\mathrm{I}_{\mathrm{p}}-\overline{\mathbf{L}}(x)\right)^{-1}\left(\mathbf{y}_{1} \overline{\mathbf{l}_{c}}(x)+\overline{\mathbf{b}}(x)\right) .
$$

Inserting this solution into Equation (22) gives

$$
\mathbf{y}_{1}(x)=\frac{\overline{\mathbf{l}_{r}}(x)\left(\mathrm{I}_{\mathrm{p}}-\overline{\mathbf{L}}(x)\right)^{-1} \overline{\mathbf{b}}(x)+\mathbf{b}_{1}(x)}{1-\mathbf{l}_{11}(x)-\overline{\mathbf{l}_{r}}(x)\left(\mathrm{I}_{\mathrm{p}}-\overline{\mathbf{L}}(x)\right)^{-1} \overline{\mathbf{l}_{c}}(x)} .
$$

We finally obtain

$$
\overline{\mathbf{y}}(x)=\left(\mathrm{I}_{\mathrm{p}}-\overline{\mathbf{L}}(x)\right)^{-1}\left(\frac{\overline{\mathbf{l}_{r}}(x)\left(\mathrm{I}_{\mathrm{p}}-\overline{\mathbf{L}}(x)\right)^{-1} \overline{\mathbf{b}}(x)+\mathbf{b}_{1}(x)}{1-\mathbf{l}_{11}(x)-\overline{\mathbf{l}_{r}}(x)\left(\mathrm{I}_{\mathrm{p}}-\overline{\mathbf{L}}(x)\right)^{-1} \overline{\mathbf{l}_{c}}(x)} \cdot \overline{\mathbf{l}_{c}}(x)+\overline{\mathbf{b}}(x)\right) .
$$

Set $\gamma(x)=\mathbf{l}_{11}(x)+\overline{\mathbf{l}_{r}}(x)\left(\mathrm{I}_{\mathrm{p}}-\overline{\mathbf{L}}(x)\right)^{-1} \overline{\mathbf{l}_{c}}(x)$ and define $\mathbf{k}(x)$ by

$$
\mathbf{k}_{1}(x)=\overline{\mathbf{l}_{r}}(x)\left(\mathrm{I}_{\mathrm{p}}-\overline{\mathbf{L}}(x)\right)^{-1} \overline{\mathbf{b}}(x)+\mathbf{b}_{1}(x),
$$

and

$$
\overline{\mathbf{k}}(x)=\left(\mathrm{I}_{\mathrm{p}}-\overline{\mathbf{L}}(x)\right)^{-1}\left(\left(\overline{\mathbf{l}_{r}}(x)\left(\mathrm{I}_{\mathrm{p}}-\overline{\mathbf{L}}(x)\right)^{-1} \overline{\mathbf{b}}(x)+\mathbf{b}_{1}(x)\right) \cdot \overline{\mathbf{l}_{c}}(x)+(1-\gamma(x)) \cdot \overline{\mathbf{b}}(x)\right) .
$$

Then we have

$$
\mathbf{y}(x)=\frac{\mathbf{k}(x)}{1-\gamma(x)} .
$$

Note, that $\mathbf{k}(x)$ is analytic for $x$ in a neighborhood of $x_{0}$. Note furthermore, that $\gamma(x)$ is also analytic for $x$ in a neighborhood of $x_{0}$ and that it is a positive function, and thus, a monotonically increasing function (again, this can be shown with the help of the Neumann series). We also know that $\gamma\left(x_{0}\right)=1$ since otherwise $\left(\mathrm{I}_{\mathrm{p}}-\mathbf{L}\left(x_{0}\right)\right)$ would be an invertible operator (contrary to $r\left(\mathbf{L}\left(x_{0}\right)\right)=1$ ). Finally we set (for $\left.x \neq x_{0}\right)$

$$
\mathbf{f}(x)=\frac{x_{0}-x}{1-\gamma(x)} \cdot \frac{\mathbf{k}(x)}{x_{0}} .
$$

We obtain that

$$
\mathbf{y}(x)=\frac{1}{1-\frac{x}{x_{0}}} \mathbf{f}(x) .
$$

In order to finish the proof of Theorem 4 for $\mathbf{v}=\mathbf{0}$ it suffices to show that $\mathbf{f}(x)$ can be analytically continued to $x_{0}$ and that $\mathbf{f}\left(x_{0}\right)_{j} \neq 0$ for all $j$. First note that

$$
\lim _{x \rightarrow x_{0}} \mathbf{f}(x)=\lim _{x \rightarrow x_{0}} \frac{x_{0}-x}{\gamma\left(x_{0}\right)-\gamma(x)} \cdot \frac{\mathbf{k}(x)}{x_{0}}=\frac{1}{\gamma^{\prime}\left(x_{0}\right)} \cdot \frac{\mathbf{k}\left(x_{0}\right)}{x_{0}} .
$$


This implies that for every $\ell \in \ell^{q}$ the limit

$$
\lim _{x \rightarrow x_{0}} \ell \cdot \mathbf{f}(x)
$$

exists. Riemann's theorem on removable singularities now implies that $\boldsymbol{\ell} \cdot \mathbf{f}(x)$ can be continued analytically to $x_{0}$ for all $\boldsymbol{\ell} \in \ell^{q}$ which finally implies that $\mathbf{f}(x)$ can be analytically continued to $x_{0}$. Since $\gamma^{\prime}\left(x_{0}\right) \neq 0$ ( $\gamma$ is positive) and $\mathbf{k}\left(x_{0}\right)_{j} \neq 0$ for all $j$ (this follows from irreducibility with the help of the Neumann series), we have also proved that $\mathbf{f}\left(x_{0}\right)_{j} \neq 0$ for all $j$.

In the second part of the proof we show that the result holds also true for $\mathbf{v}$ in a neighborhood of $\mathbf{0}$. First we see that Equation (23) can also be solved with the additional parameter $\mathbf{v}$. Indeed, the analyticity of $\mathbf{F}$ and Lemma 4 imply that $r\left(\mathbf{L}\left(x_{0}, \mathbf{v}\right)\right)<1$ for all $\mathbf{v}$ in a neighborhood of $\mathbf{0}$. Inserting this solution into Equation 22, we obtain in the same way as above that

$$
\mathbf{y}(x, \mathbf{v})=\frac{\mathbf{k}(x, \mathbf{v})}{1-\gamma(x, \mathbf{v})} .
$$

for some analytic function $\mathbf{k}(x, \mathbf{v})$ and for $\gamma(x, \mathbf{v})=\mathbf{l}_{11}(x, \mathbf{v})+\overline{\mathbf{l}_{r}}(x, \mathbf{v})\left(\mathrm{I}_{\mathrm{p}}-\overline{\mathbf{L}}(x, \mathbf{v})\right)^{-1} \overline{\mathbf{l}_{c}}(x, \mathbf{v})$. Since $\gamma^{\prime}\left(x_{0}, \mathbf{0}\right)>0$, the implicit function theorem implies that there exists an analytic function $x_{0}(\mathbf{v})$ in a neighborhood of $\mathbf{0}$ such that

$$
\gamma\left(x_{0}(\mathbf{v}), \mathbf{v}\right)=1 .
$$

Thus we obtain with

$$
\mathbf{f}(x)=\frac{x_{0}(\mathbf{v})-x}{1-\gamma(x, \mathbf{v})} \cdot \frac{\mathbf{k}(x, \mathbf{v})}{x_{0}(\mathbf{v})}, \quad x \neq x_{0}(\mathbf{v})
$$

that

$$
\mathbf{y}(x, \mathbf{v})=\frac{1}{1-\frac{x}{x_{0}(\mathbf{v})}} \mathbf{f}(x, \mathbf{v}) .
$$

As before, we see that $\mathbf{f}$ can be continued analytically to $x_{0}(\mathbf{v})$. This finally proves Theorem 4 